\title{
Ectopic upregulation of membrane-bound IL6R drives vascular remodeling in pulmonary arterial hypertension
}

\author{
Yuichi Tamura, Carole Phan, Ly Tu, Morane Le Hiress, Raphaël Thuillet, Etienne-Marie Jutant, Elie Fadel, Laurent Savale, \\ Alice Huertas, Marc Humbert, and Christophe Guignabert
}

INSERM UMR_S 999, Hôpital Marie Lannelongue, Le Plessis-Robinson, France; Faculté de Médecine, Univ. Paris-Sud, Université Paris-Saclay, Le Kremlin-Bicêtre, France; Assistance Publique-Hôpitaux de Paris (AP-HP), Service de Pneumologie, Centre de Référence de l'Hypertension Pulmonaire, DHU Thorax Innovation, Hôpital Bicêtre, Le Kremlin-Bicêtre, France.

\begin{abstract}
Pulmonary arterial hypertension (PAH) is characterized by a progressive accumulation of pulmonary artery smooth muscle cells (PA-SMCs) in pulmonary arterioles leading to the narrowing of the lumen, right heart failure, and death. Although most studies have supported the notion of a role for IL-6/glycoprotein 130 (gp130) signaling in PAH, it remains unclear how this signaling pathway determines the progression of the disease. Here, we identify ectopic upregulation of membrane-bound IL-6 receptor (IL6R) on PA-SMCs in PAH patients and in rodent models of pulmonary hypertension (PH) and demonstrate its key role for PA-SMC accumulation in vitro and in vivo. Using Sm22a-Cre II6rfl/fl, which lack II6r in SM22A-expressing cells, we found that these animals are protected against chronic hypoxia-induced PH with reduced PA-SMC accumulation, revealing the potent pro-survival potential of membrane-bound IL6R. Moreover, we determine that treatment with IL6R-specific antagonist reverses experimental PH in two rat models. This therapeutic strategy holds promise for future clinical studies in $\mathrm{PAH}$.
\end{abstract}

\section{Introduction}

Despite the recent development of new therapeutic agents (1), therapies against pulmonary arterial hypertension (PAH) remain palliative, and most patients still die from the disease or fail to respond adequately to medical therapy, with a 5 -years survival of $59 \%$ (2). Despite the progress made in our understanding of the pathological changes associated with PAH in pulmonary arteries, lung transplantation is often necessary. Thus, targeting the progressive aberrant accumulation of pulmonary artery smooth muscle cells (PA-SMCs) within the pulmonary vascular wall of small distal arteries, the key factor of $\mathrm{PAH}$ pathogenesis, is necessary for effective PAH therapy.

Since substantially elevated circulating levels of certain inflammatory mediators, including IL- 6 , have been found to correlate with worse clinical outcomes, quality of life-related symptoms, and/or death in PAH patients (3-5), the sustained inflammation in PAH has moved from a common histopathological finding to a potential contributor to the perpetuation of the disease. However, the field is currently lacking a mechanistic vantage point to understand how inflammation, and in particular IL-6-dependent signaling, contributes to the disease pathogenesis, a prerequisite for the development of novel therapeutic strategies.

\section{Related Commentary: p. 1720}

Authorship note: YT and CP contributed equally to this work.

Conflict of interest: MH and LS report grants, personal fees, and nonfinancial support received in the past 3 years from Actelion, Pfizer, Bayer, GlaxoSmithKline, and MSD, outside the scope of the submitted work.

Submitted: July 24, 2017; Accepted: February 8, 2018.

Reference information: / Clin Invest. 2018;128(5):1956-1970.

https://doi.org/10.1172/JCI96462.
Two different signaling pathways for IL-6 have been described (6). In the classical signaling (cis) pathway, IL-6 binds to membrane-bound IL6R (CD126), leading to dimerization and activation of the signal-transducing protein gp130. IL6R expression is restricted to hepatocytes, neutrophils, monocytes/ macrophages, and some lymphocytes. On the other hand, in the trans-signaling pathway, IL-6 binds to soluble IL6R (sIL6R) and activates gp130. The trans-signaling pathway has some important biological effects, such as growth and survival of premalignant cells or resident tissue cells $(7,8)$. In particular, consideration of this pathway enlarges the spectrum of targets for IL-6, because cells that do not express membrane-bound IL6R can still be stimulated by the IL-6/sIL6R complex. Importantly, the delivery of recombinant IL- 6 protein in rodents has been shown to be sufficient to cause pulmonary vascular remodeling and pulmonary hypertension $(\mathrm{PH})$ or to exaggerate the pulmonary hypertensive response to chronic hypoxia $(9,10)$. Furthermore, IL-6-overexpressing mice spontaneously develop PH and pulmonary vascular remodeling, whereas Il6-knockout mice are more resistant to the development of $\mathrm{PH}$ induced by chronic hypoxia $(11,12)$. In this context, although blockade of IL-6 signaling represents a promising target $(3,4,9-14)$, it remains unclear how this signaling pathway determines the progression and ultimately the severity of the disease, and it is therefore difficult to develop therapeutic strategies.

Herein, we investigated (a) whether IL-6/gp130 signaling contributes directly to the progressive accumulation of pulmonary vascular cells within walls of pulmonary arteries in experimental and human PAH, a hallmark of the disease; and (b) the efficacy and the therapeutic implications of blockade of IL-6/gp130 signaling in experimental $\mathrm{PH}$. 
A
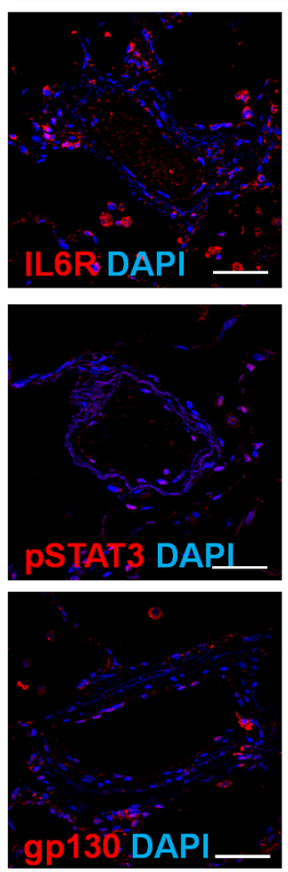

Control
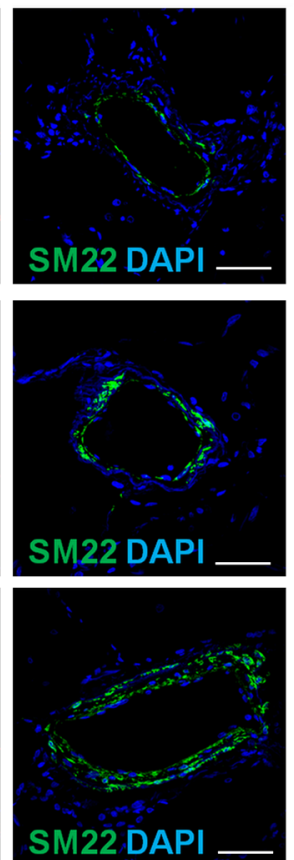
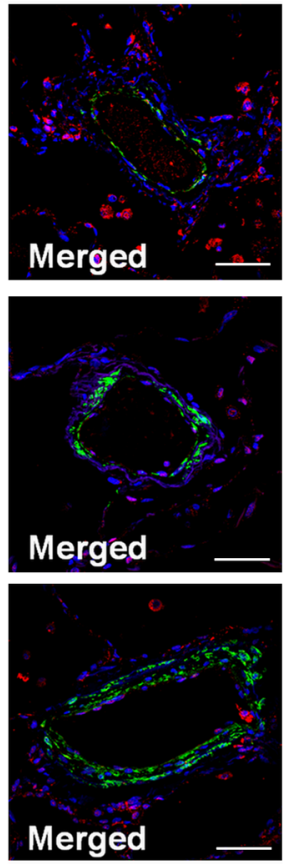

Idiopathic PAH
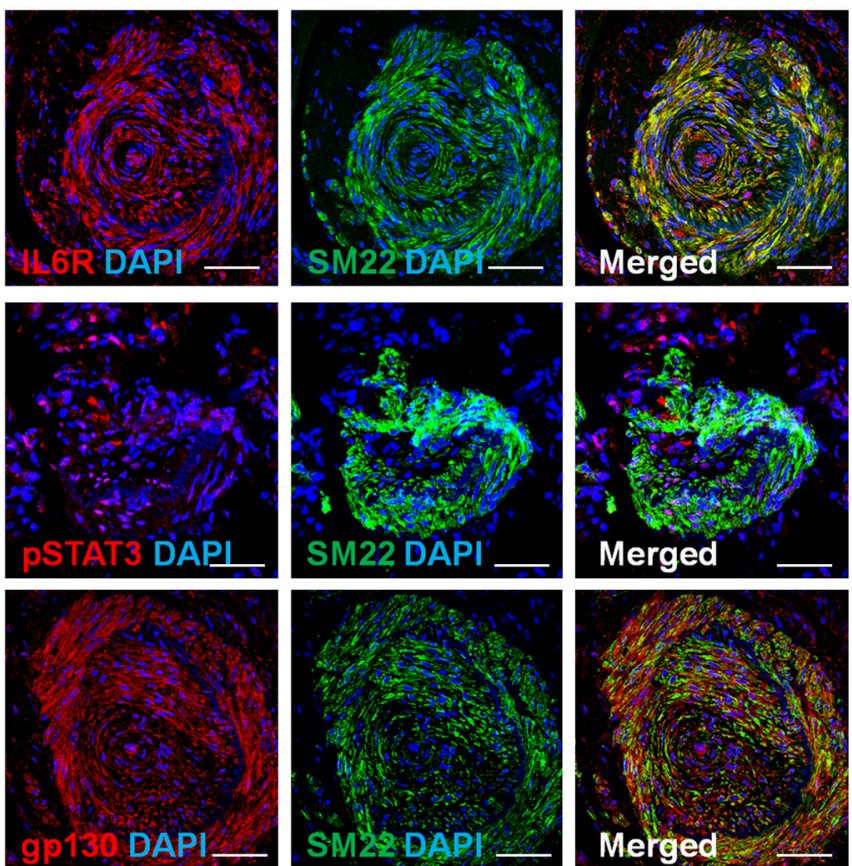

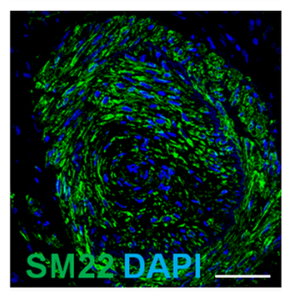

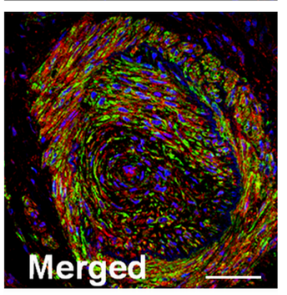

B

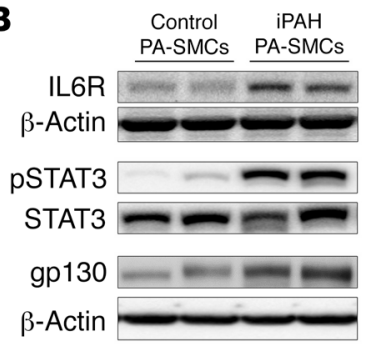

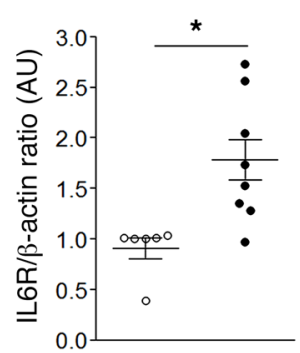
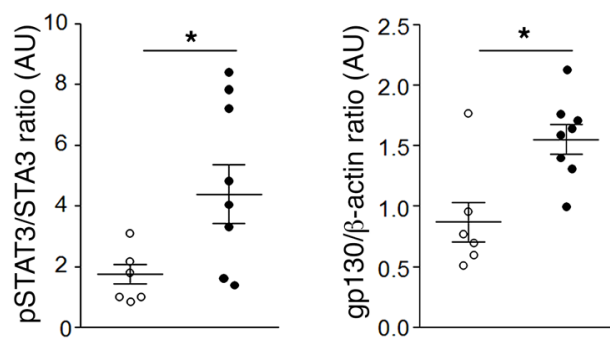

O Control PA-SMCs

- iPAH PA-SMCs
C

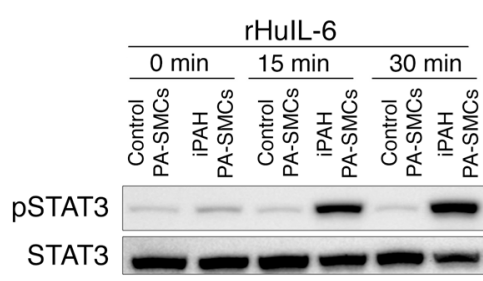

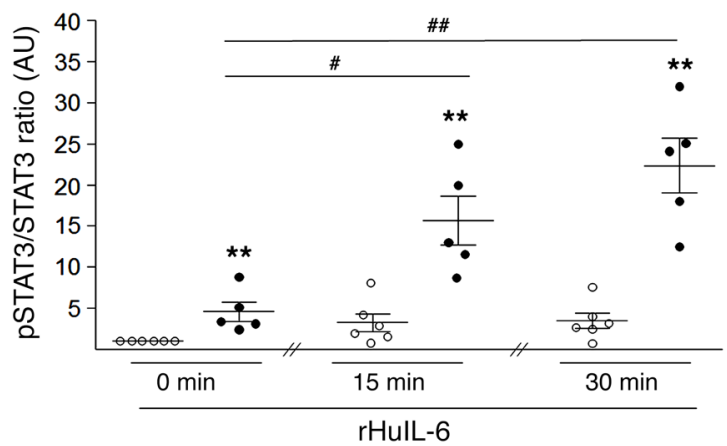

Figure 1. Upregulation of IL-6 cis- and trans-signaling pathways in PA-SMCs and lungs of patients with iPAH as compared with control patients. (A) Double staining with IL6R, pSTAT3, or gp130 with SM22 and DAPI in lungs from control subjects and iPAH patients $(n=7)$. (B) Representative Western blots and quantification of IL6R/ $\beta$-actin, pSTAT3/STAT3, and gp130/ $\beta$-actin ratios in human pulmonary PA-SMCs derived from control subjects and iPAH patients $(n=6-8)$. (C) Representative Western blots and quantification of PSTAT3/STAT3 ratio in human pulmonary PA-SMCs derived from control subjects and IPAH patients exposed for 0,15 , or 30 minutes to rHulL-6 protein $(n=5-6)$. Scale bars: $50 \mu \mathrm{m}$. Data are presented as mean \pm SEM. Comparisons were made using the nonparametric Mann-Whitney $U$ test or 2 -way ANOVA with Bonferroni's post hoc tests. ${ }^{*} P<0.05,{ }^{* *} P<0.01$ versus control PA-SMCs and versus control PA-SMCs at 0 minutes; ${ }^{\#} P<0.05,{ }^{\# \#} P<0.01$ versus iPAH PA-SMCs at 0 minutes.

\section{Results}

Upregulation of IL-6 cis- and trans-signaling pathways in PA-SMCs and lungs of patients with idiopathic PAH. We first sought to specifically investigate the global expression pattern of key actors in IL-6 signaling in lung specimens obtained during lung transplan- tation in idiopathic PAH (iPAH) patients and during lobectomy or pneumonectomy for localized lung cancer in control individuals. Our confocal microscopic analyses demonstrated an unexpected strong ectopic upregulation of membrane-bound IL6R in the smooth muscle layer of remodeled distal pulmonary arteries from 
Table 1. Characteristics of controls, iPAH, and hPAH patients before lung transplantation, and levels of circulating IL-6, sIL6R, and soluble gp130 proteins

\begin{tabular}{|c|c|c|c|}
\hline & IPAH & hPAH & Controls \\
\hline$n$ & 23 & 15 & 17 \\
\hline Age, yr & $50.9 \pm 2.6$ & $49.7 \pm 3.3$ & $40.2 \pm 3.3$ \\
\hline Sex, M/F & $5 / 18$ & $6 / 9$ & $8 / 9$ \\
\hline Mutations in BMPR2 gene, $n$ & 0 & 15 & NA \\
\hline \multicolumn{4}{|l|}{ NYHA functional class, $n$} \\
\hline Class I & 5 & 3 & NA \\
\hline Class II & 11 & 7 & NA \\
\hline Class III & 7 & 5 & NA \\
\hline Class IV & 0 & 0 & NA \\
\hline BNP, ng/l & $66 \pm 21$ & $90 \pm 37$ & \\
\hline 6-MWD, m & $450 \pm 19$ & $462 \pm 20$ & NA \\
\hline mPAP, mmHg & $44.3 \pm 3.1$ & $50.3 \pm 2.0$ & NA \\
\hline $\mathrm{Cl}, \mathrm{l} / \mathrm{min} / \mathrm{m}^{2}$ & $2.8 \pm 0.2$ & $2.4 \pm 0.2$ & NA \\
\hline PVR, WU & $6.5 \pm 0.6$ & $9.2 \pm 1.2$ & NA \\
\hline \multicolumn{4}{|l|}{ Specific PAH therapy } \\
\hline ERA + PDE5i + prostanoids & 4 & 5 & NA \\
\hline ERA + PDE5i & 8 & 7 & NA \\
\hline ERA + prostanoids & 1 & 1 & NA \\
\hline PDE5i + prostanoids & 4 & 1 & NA \\
\hline ERA & 4 & 0 & NA \\
\hline PDE5i & 1 & 1 & NA \\
\hline $\mathrm{Ca}^{2+}$ blocker & 1 & 0 & NA \\
\hline Circulating IL-6 levels, pg/ml & $51 \pm 24.6^{A}$ & $187 \pm 52.2^{A, B}$ & $1.2 \pm 0.3$ \\
\hline Circulating sIL6R levels, ng/ml & $51 \pm 2.5^{A}$ & $42 \pm 3.8$ & $33 \pm 2.1$ \\
\hline Circulating sgp130 levels, ng/ml & $328 \pm 12.0$ & $311 \pm 11.3$ & $298 \pm 9.4$ \\
\hline
\end{tabular}

Values are mean \pm SEM. 6-MWD, 6-minute walk test; BNP, brain natriuretic peptide; $\mathrm{Cl}$, cardiac index; ERA, endothelin receptor antagonist; NA, not applicable; NYHA, New York Heart Association; PVR, pulmonary vascular resistance; PDE5i, phosphodiesterase 5 inhibitors; R, receptor; sgp130, soluble gp130; sIL6R, soluble IL-6 receptor; WU, Wood units. ${ }^{A}$ Statistical significance compared with controls. ${ }^{B}$ Statistical significance compared with iPAH (ANOVA by Kruskal-Wallis test and Dunn's correction). compared with control PA-SMCs, clearly indicating that this ectopic IL6R upregulation amplified the cellular responses to extracellular stimulation of exogenous IL-6 (Figure 1C).

Ectopic IL6R upregulation contributes to PA-SMC survival in $P$ PAH. Since IL-6 and STAT3 can directly regulate many genes implicated in cell survival and cell proliferation $(15,16)$, we next investigated the functional importance of IL6R in PA-SMCs and assessed whether this ectopic IL6R upregulation could serve as a pro-survival mechanism in $\mathrm{PAH}$ PA-SMCs, thereby contributing to increased PA-SMC accumulation, one of the hallmarks of the disease.

Indeed, we found that rHuIL-6 inhibited the activation of the executioner caspases caspase- 3 and caspase-7 induced by staurosporine, cycloheximide, and TNF- $\alpha$ in iPAH PA-SMCs, in contrast to control PA-SMCs (Figure 2A). Furthermore, we found that rHuIL- 6 reduced the percentage of TUNEL-positive iPAH PA-SMCs induced by staurosporine by about 3-fold (Figure 2B). Consistent with this finding, increased expression of the antiapoptotic proteins MCL-1 and BCL2 and a high BCL2/Bax ratio were found in $\mathrm{PAH}$ PA-SMCs exposed to rHuIL-6 (Figure 2C).

Transfection of PA-SMCs with an IL6R expression plasmid ( $p C M V 3-I L 6 R-G F P S p a r k$ ) has a strong survival-promoting effect (Figure $3, \mathrm{~A}-\mathrm{C}$ ). Indeed, the overactivation of the IL-6/IL6R axis in these cells overexpressing the IL6R markedly reduced the activation of caspase- $3 / 7$ induced by staurosporine by more than 2 -fold and decreased the percentage of TUNEL-positive cells induced by staurosporine when compared with iPAH PA-SMCs transfected with the control plasmid ( $p C M V 3-C$-GFPSpark)

(Figure 3, B and C). Consistent with these obser-

patients with iPAH, compared with controls (Figure 1A). Using 2 different antibodies that specifically target 2 distinct IL6R loci, we confirmed that this ectopically upregulated membrane-bound IL6R was the transmembrane form of the receptor (Supplemental Figure 1A). Of note, weak staining within the dysfunctional pulmonary endothelium was found. Interestingly, double labeling with IL6R, phosphorylated STAT3 (Tyr705), or gp130 and the smooth muscle-specific SM22 protein demonstrated substantial overactivation of IL-6/gp130 signaling in PA-SMCs from remodeled vessels of patients with iPAH; this supports the hypothesis that IL-6 can signal via the IL-6 classical (cis) or IL-6 trans-signaling pathway in PAH (Figure 1A, Table 1, and Supplemental Figure 1A).

Consistent with these in situ observations, we found that freshly isolated human PA-SMCs from iPAH patients also exhibited marked upregulation of membrane-bound IL6R protein expression, accompanied by strong STAT3 activation and higher expression of gp130, when compared with control cells, outside of their natural in vivo environment (Figure 1B). This aberrant IL6R upregulation was further corroborated by the demonstration that primary cultures of iPAH PA-SMCs exhibited stronger elevation of pSTAT3 when exposed to recombinant human IL-6 (rHuIL-6) vations, parallel evaluations showed that transfecting PA-SMCs with the IL6R expression plasmid substantially increased expression of the antiapoptotic proteins MCL-1 and BCL2 and the BCL2/Bax ratio in contrast to iPAH PA-SMCs transfected with the control plasmid (Figure 3A).

Subsequent studies were therefore carried out using a specific neutralizing antibody directed against IL6R (anti-IL6R) and the nonpeptide IL6R/sIL6R antagonist ERBF (also called 20S,21epoxy-resibufogenin-3-formate) (17-19) to determine whether blocking the IL6R in IPAH PA-SMCs would result in suppression of the apoptosis-resistant PA-SMC phenotype induced by rHuIL-6. We obtained evidence showing that anti-IL6R and ERBF partially abolished the apoptotic response of iPAH PA-SMCs to staurosporine when compared with vehicle-treated cells, as assessed by caspase-3/7 activity assay and TUNEL staining (Figure 4, A and B). Consistent with these observations, we found a substantial attenBax ratio in iPAH PA-SMCs exposed to rHuIL- 6 when treated with anti-IL6R and IL6R/sIL6R antagonist (Figure 4C). Of note, we found no effect of exogenous rHuIL-6 on iPAH PA-SMC proliferation, consistent with the literature (20) (Supplemental Figure 1B). uation in MCL-1 and BCL2 protein levels as well as in the BCL2/ 
A
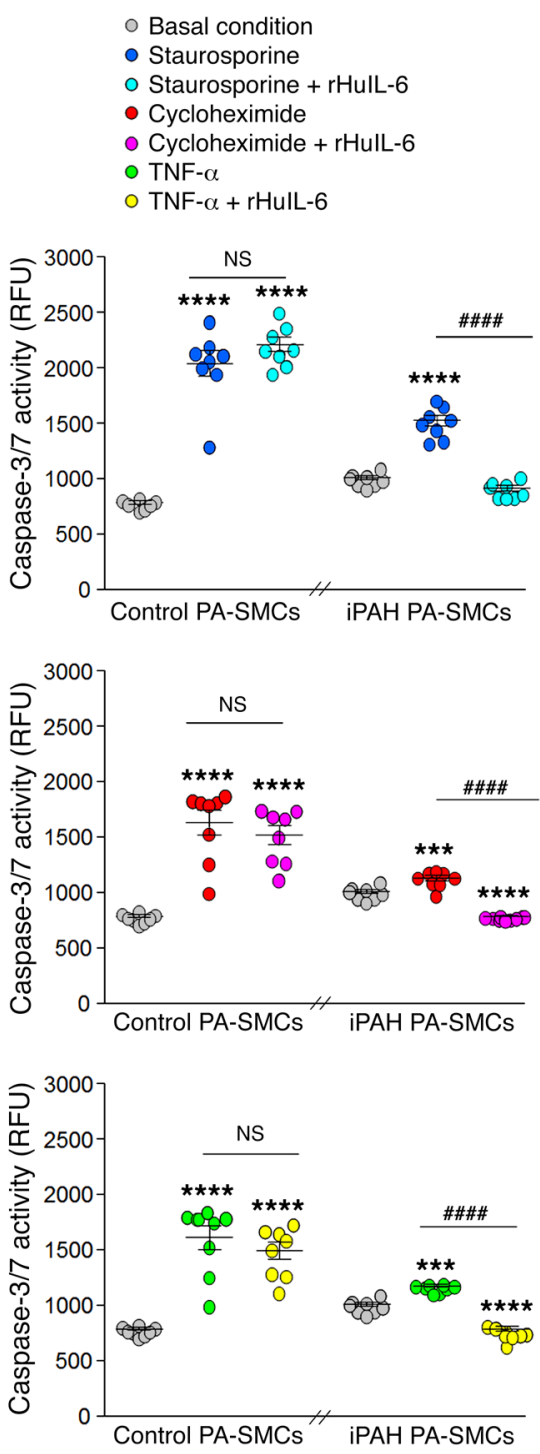

B

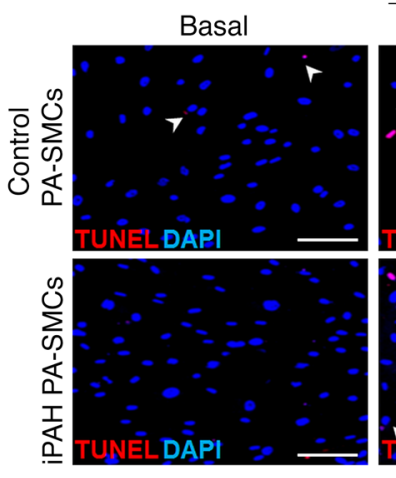

Control PA-SMCs
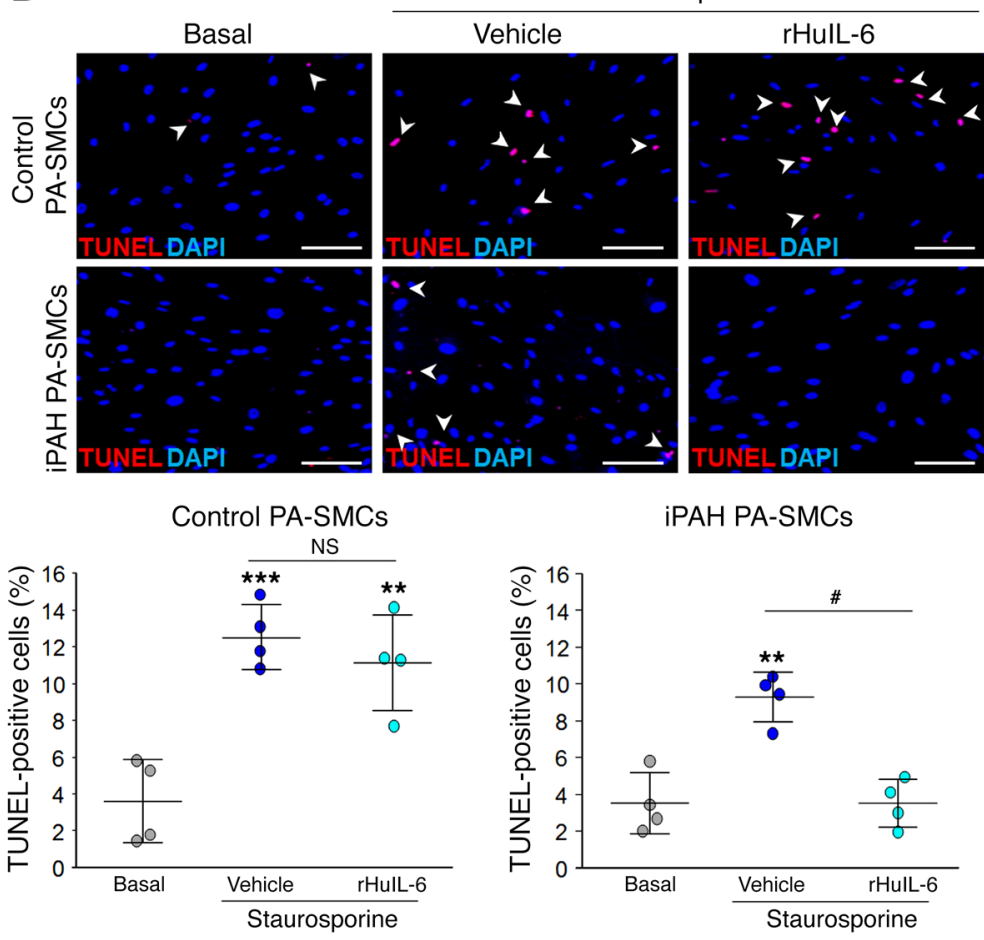
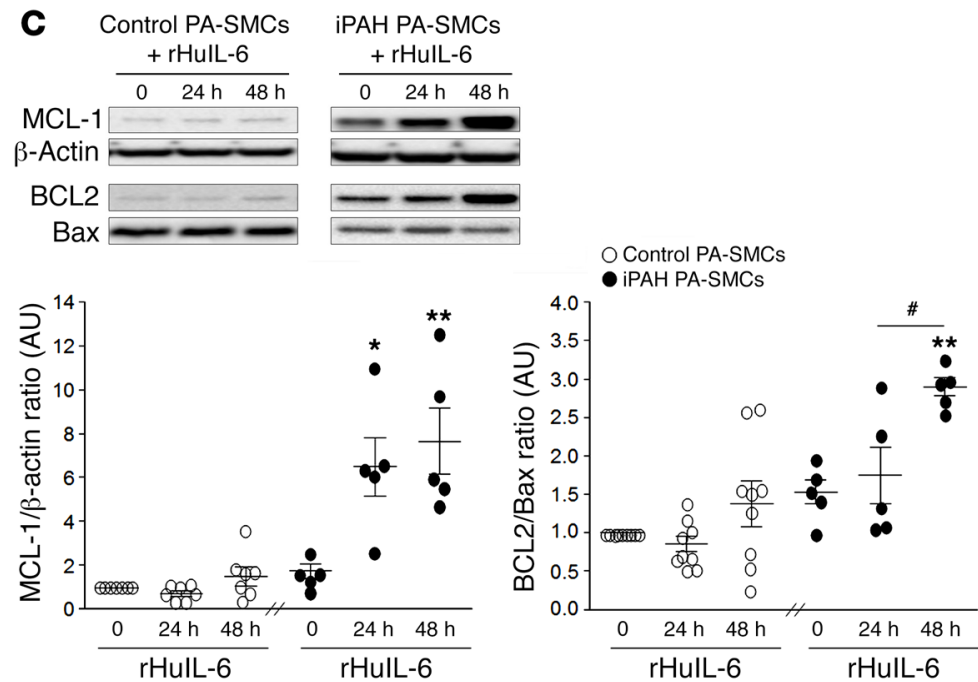

Figure 2. rHulL-6 exaggerates the apoptosis-resistant phenotype in iPAH PA-SMCs. (A) Caspase-3/7 activity in PA-SMCs derived from control and iPAH patients following staurosporine, cycloheximide, or TNF- $\alpha$ treatment in the presence or absence of rHulL-6 $(n=7-8)$. (B) Representative TUNEL images and quantification of the percentage of TUNEL-positive cells (arrowheads) in control and IPAH PA-SMCs exposed to staurosporine in the presence or absence of rHulL-6 $(n=4)$. (C) Representative Western blots and quantification of MCL-1/ $\beta$-actin and BCL2/Bax ratios in human PA-SMCs derived from control and IPAH at different time points following exposure to rHulL-6 $(n=5-9)$. Scale bars: $50 \mu \mathrm{m}$. Data are presented as mean \pm SEM. Comparisons were made using 2-way ANOVA with Bonferroni's post hoc tests. ${ }^{*} P<0.05,{ }^{* *} P<0.01,{ }^{* *} P<0.001$; ${ }^{* * *} P<0.0001$ versus PA-SMCs under basal conditions or

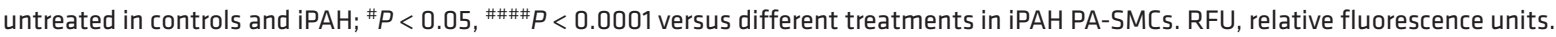

Interestingly, we reduced the STAT3 activation state by about $60 \%$ using either anti-IL6R or IL6R/sIL6R antagonist in iPAH PA-SMCs exposed to rHuIL- 6 as compared with vehicle ( $P<0.05$ for both) (Supplemental Figure 1, C and D). Since gp130 is shared by cytokines other than IL-6, including IL-11 and leukemia inhibitory factor (LIF), we validated that this inhibition of the IL-6 signaling pathway by ERBF is specific and does not interact with IL-11 and LIF downstream signaling (Supplemental Figure 1, D-F).
Transgenic mice deficient in IL6R in the smooth muscle are protected against the development of experimental $\mathrm{PH}$. Next, to better understand the functional importance in the pathogenesis of $\mathrm{PH}$ of this ectopic membrane-bound IL6R upregulation in PA-SMCs, we used $S m 22 a$-Cre Il6rflfl mice, in which the Il6r gene is deleted in SM22-expressing cells (represented in the figure labels as -/-) (Figures 5 and 6, and Supplemental Figure 2, A-C). Interestingly, the Sm22a-Cre Il6rfl/fl mice exposed to hypoxia (10\% fraction 
A

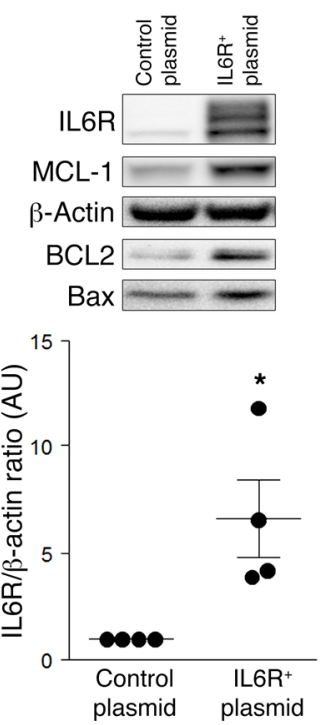

B
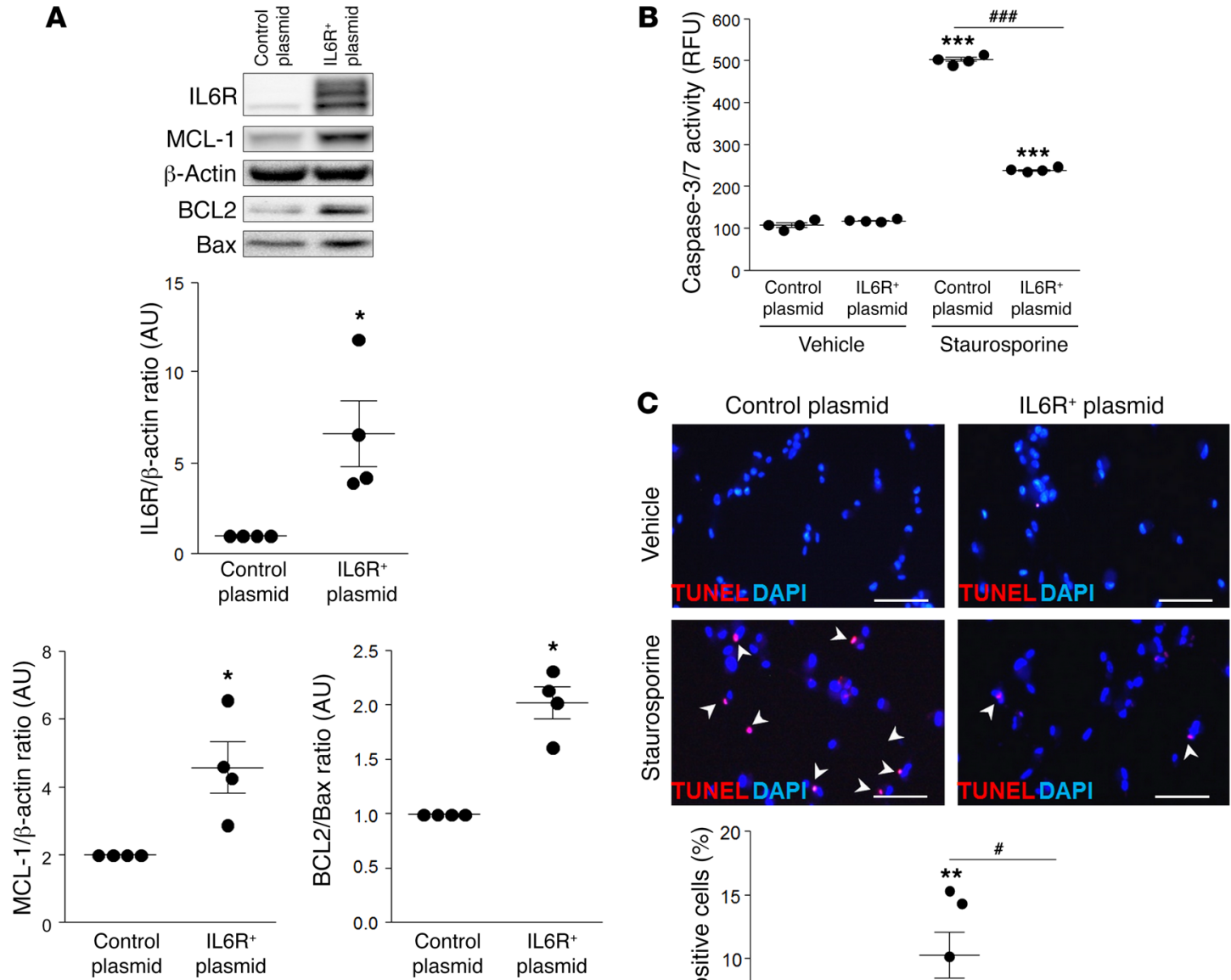
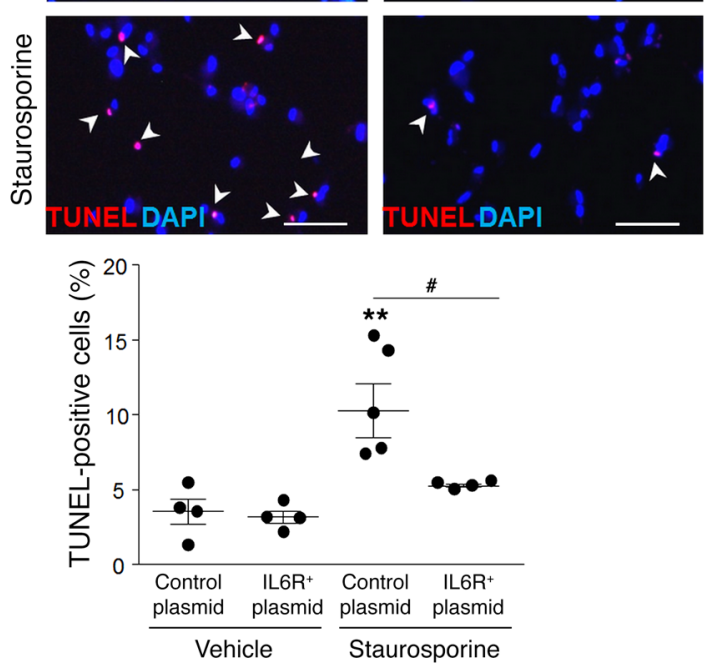

Figure 3. Membrane-bound IL6R serves as a pro-survival mechanism in iPAH PA-SMCs. (A) Representative Western blots and quantification of IL6R/ $\beta$-actin, MCL-1/ $\beta$-actin, and BCL2/Bax ratios in PA-SMCs transfected with either an IL6R expression plasmid (pCMV3-IL6R-GFPSpark) or a control plasmid ( $p$ CMV3-C-GFPSpark) $(n=4)$. (B) Effects of IL6R overexpression on caspase-3/7 activity $(n=4)$ and $(C)$ percentage of TUNEL-positive cells (arrowheads) in PA-SMCs derived iPAH patients $(n=4-5)$. Scale bars: $50 \mu \mathrm{m}$. Comparisons were made using the nonparametric Mann-Whitney $U$ test or 2-way ANOVA with Bonferroni's post hoc tests. ${ }^{*} P<0.05,{ }^{* *} P<0.01,{ }^{* *} P<0.001$ versus PA-SMCs transfected with the control plasmid; ${ }^{\#} P<0.05$, \#\#\# $P<0.001$ versus PA-SMCs transfected with the control plasmid and exposed to staurosporine.

of inspired oxygen $\left.\left[\mathrm{FiO}_{2}\right]\right)$ for 3 weeks were less susceptible to the development of chronic hypoxia-induced PH than Sm22a-Cre $\mathrm{Il}^{\mathrm{ll/+}}(+/-)$ or Sm22a-Cre Il6r $r^{+++}(+/+)$littermate mice $(P<0.01)$. The severity of the disease was assessed by right ventricular systolic pressure (RVSP) measurement (Figure 5A), by right ventricular hypertrophy (RVH) (Figure 5B), and by pulmonary distal arterial muscularization (Figure $5 \mathrm{C}$ ). We also noted that under hypoxic conditions, collagen deposition and the degree of inflammatory cell infiltration were significantly decreased in lungs from $\mathrm{Sm} 22 a$ -

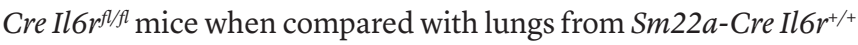
mice (Supplemental Figure 2D).

Consistent with our findings, a lower STAT3 activation state, together with decreased expression of MCL-1 and BCL2, was observed in lungs from Sm22a-Cre Il6r/f/l mice exposed to chronic hypoxia when compared with hypoxic WT mice (Figure 6, A and B). Importantly, a greater apoptotic response was measured in lungs from $\mathrm{Sm} 22 \mathrm{a}$-Cre $\mathrm{Il} 6 \mathrm{r}^{\mathrm{fl} / \mathrm{l}}$ mice exposed to chronic hypoxia when compared with hypoxic WT mice, as assessed by caspase-3/7 activity assay and TUNEL staining (Figure 6, C and D). Of note, no difference was noted in pulmonary hemodynamic or lung structure between Sm22a-Cre Il6r+/+, Sm22a-Cre Il6r $r^{+/ f}$, or Sm22a-Cre $I l 6 r^{f / / f}$ mice when exposed to room air (Figure 5).

In vivo evidence of IL6R/sIL6R antagonist efficacy in 2 complementary chronic animal models of severe PH. Based on these results, we next examined the functional importance of this ectopic membrane-bound IL6R upregulation in PA-SMCs for the pulmonary vascular remodeling in 2 well-recognized and widely used $\mathrm{PH}$ rat models: treatment with monocrotaline (MCT) (Figures 7, 8, and 9) and SU5416 combined with chronic hypoxia (SuHx) (Figures 10 and 11). In both PH rat models, we found increased circulating levels of IL-6 and sIL6R, as well as a significant increase in IL6R expression in rat PA-SMCs and total lungs (Figure 7 and Figure 10, A-C). 
A

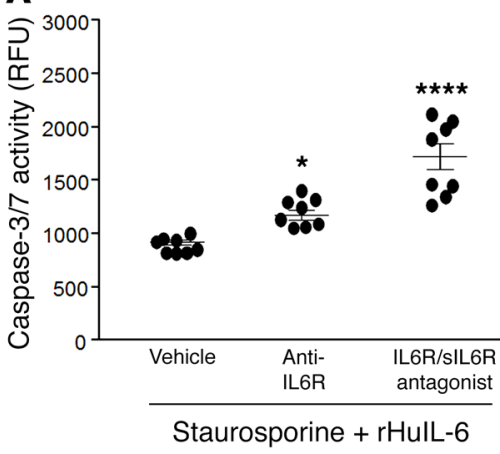

C

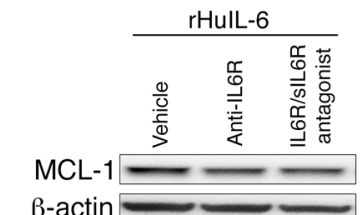

$\beta$-actin
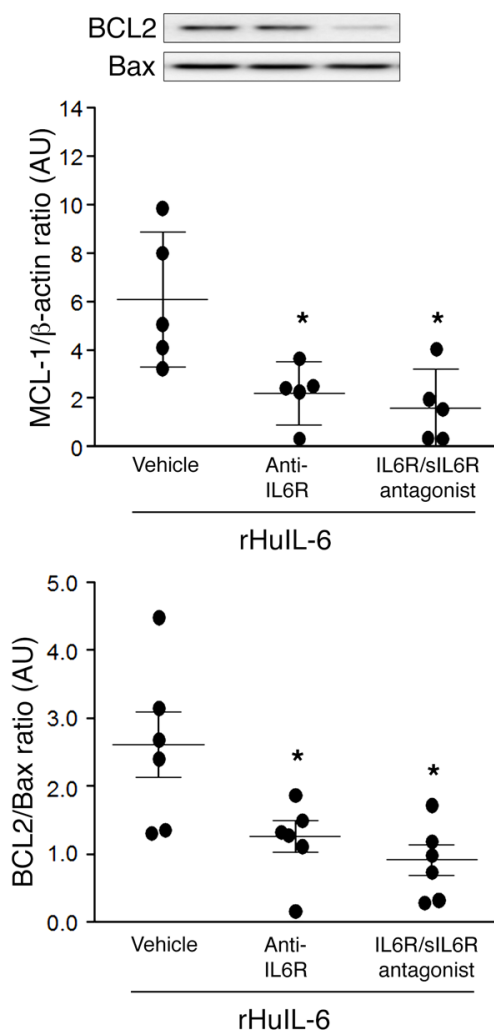

B

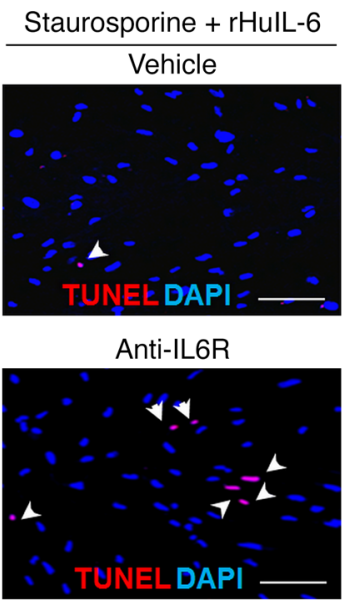

IL6R/sIL6R antagonist
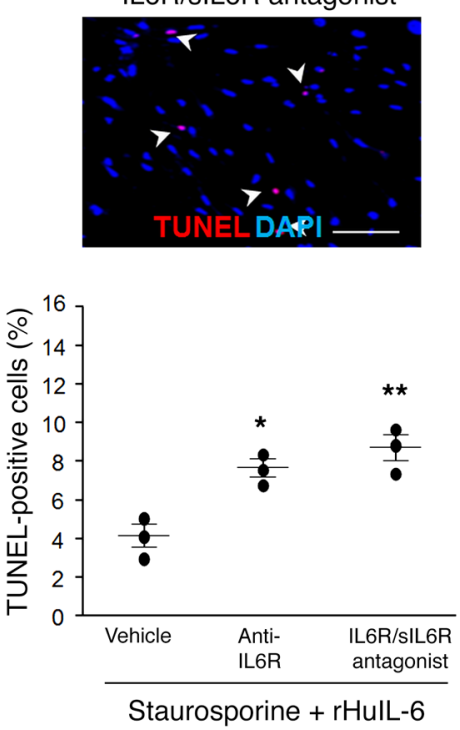

Figure 4. Effects of a specific neutralizing antibody directed against IL6R (anti-IL6R) and nonpeptide IL6R/sIL6R antagonist ERBF on survival-promoting IL6R action in PA-SMCs. (A) Caspase-3/7 activity $(n=8)$ and (B) percentage of TUNEL-positive cells (arrowheads) $(n=3)$ in iPAH PA-SMCs pretreated with rHulL-6 for 48 hours and exposed to staurosporine or vehicle. (C) Representative Western blots and quantification of MCL-1/ $\beta$-actin and BCL2/Bax ratios in iPAH PA-SMCs pretreated with rHulL-6 for 48 hours $(n=5-6)$. Scale bars: $50 \mu \mathrm{m}$. Data are presented as mean \pm SEM. Comparisons were made using 2-way ANOVA with Bonferroni's post hoc tests. ${ }^{*} P<0.05,{ }^{* *} P<0.01,{ }^{* * *} P<0.0001$ versus PA-SMCs treated with staurosporine or vehicle.

vehicle-treated animals (Figure 9A). Consistently, in both the preventive and curative protocols, we found substantial attenuation of the STAT3 activation state in the smooth muscle of MCT-injected rat treated with IL6R/sIL6R antagonist when compared with vehicle-treated rats and controls (Figure 9B). Interestingly, the beneficial effect of the IL6R/sIL6R antagonist was associated with a decrease in MCL-1 protein expression and an increase in the number of TUNEL-positive cells in lungs of MCT rats treated with IL6R/sIL6R antagonist when compared with vehicle-treated rats (Figure 9, $\mathrm{C}$ and $\mathrm{D}$ ). Consistent with findings obtained in the MCT rat PH model, we also found the IL6R/sIL6R antagonist to have curative efficacy in the second complementary rat model of severe $\mathrm{PH}(\mathrm{SuHx})$ and validated its beneficial PA-SMC proapoptotic action (Figures 10 and 11). In addition, we also noted that collagen deposition and the degree of inflammatory cell infiltration were significantly decreased in lungs and right ventricles of MCTinjected rats and SuHx rats treated with ERBF when compared with MCT and SuHx rats treated with vehicle (Supplemental Figure 3).

Interestingly, no significant cardiac effect of chronic ERBF treatment was observed in healthy rats. Indeed, echocardiographic assessment of cardiovascular function indicated no significant differences between WT rats chronically treated with ERBF and those treated with vehicle (ratio of acceleration time to ejection time [AT/ET]: $0.50 \pm 0.04$ versus $0.44 \pm$ $0.05 \mathrm{AU}, n=4-5$, NS; right ventricular ejection fraction: $83.2 \% \pm 1.9 \%$ versus $84.9 \% \pm 2.6 \%, n=4-5$, NS; CO: $341.8 \pm 30.9$ versus $260.4 \pm 19.5 \mathrm{ml} / \mathrm{min}, n=4-5$,

To test the in vivo efficacy of the nonpeptide IL6R/sIL6R antagonist ERBF, we first developed preventive and/or curative treatment protocols in the MCT rat model of PH (17) (Figure 8A). Interestingly, in both the preventive and curative protocols, daily treatment with ERBF prevented and reversed established $\mathrm{PH}$ induced by MCT as assessed by echocardiography (Figure $8 \mathrm{~B}$ ) and right heart catheterization with the measurement of mean pulmonary arterial pressure (mPAP), cardiac output (CO), total pulmonary vascular resistance (TPVR), and RVH measurement (Figure 8C). Furthermore, percent medial wall thickness and the number of muscularized distal pulmonary arteries were significantly reduced by the preventive and curative protocols in MCT- compared with
NS; respectively). This absence of cardiac effects of ERBF was confirmed by right heart catheterization (mPAP: $17.4 \pm 0.5$ versus $16.1 \pm 0.4 \mathrm{mmHg}, n=4-6$, NS; CO: $102 \pm 5$ versus $104 \pm 3 \mathrm{ml} / \mathrm{min}$, $n=4-6$, NS; respectively). Of note, no significant difference was noted in the degree of pulmonary vasoconstriction in response to acute hypoxia $\left(10 \% \mathrm{FiO}_{2}, 10\right.$ minutes) between rats chronically treated with ERBF and vehicle-treated rats ( $\triangle \mathrm{MPAP}, 4.4 \pm 0.8$ $\mathrm{mmHg}, n=3$, versus $4.8 \pm 0.7 \mathrm{mmHg}, n=3$, respectively; NS).

\section{Discussion}

High levels of serum IL-6 are associated with poor survival, quality of life-related symptoms, and/or death in patients with iPAH 
A

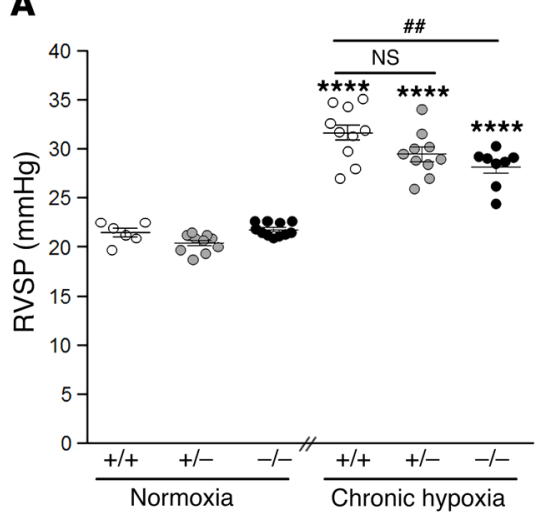

C
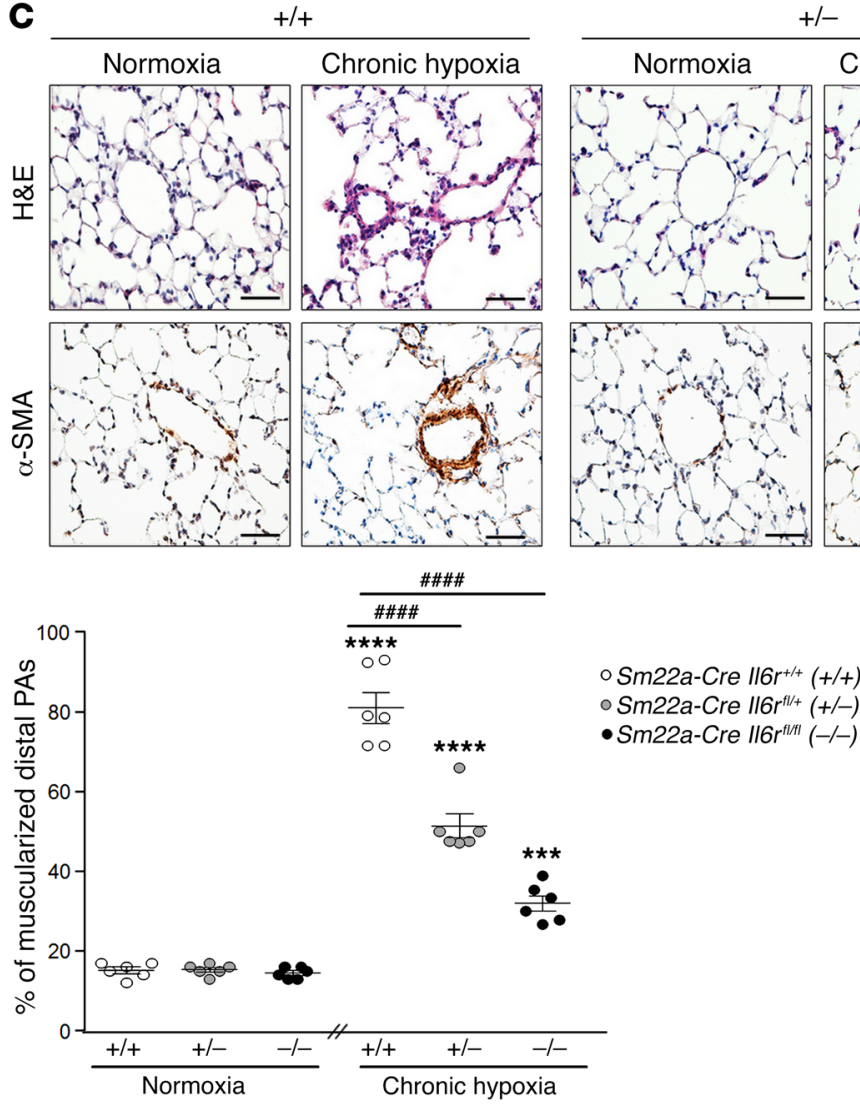

B

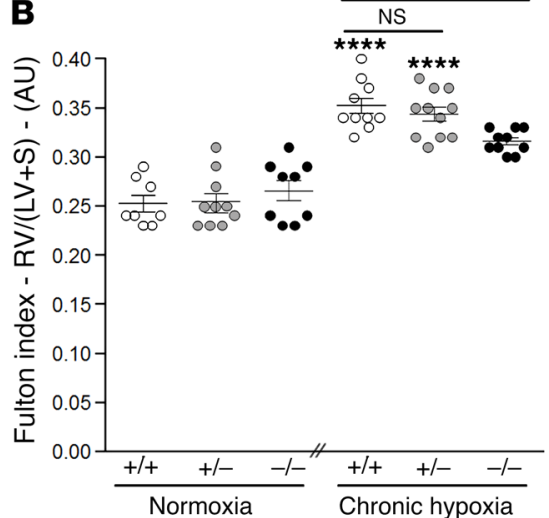

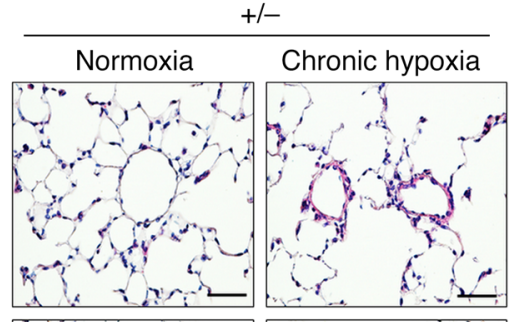
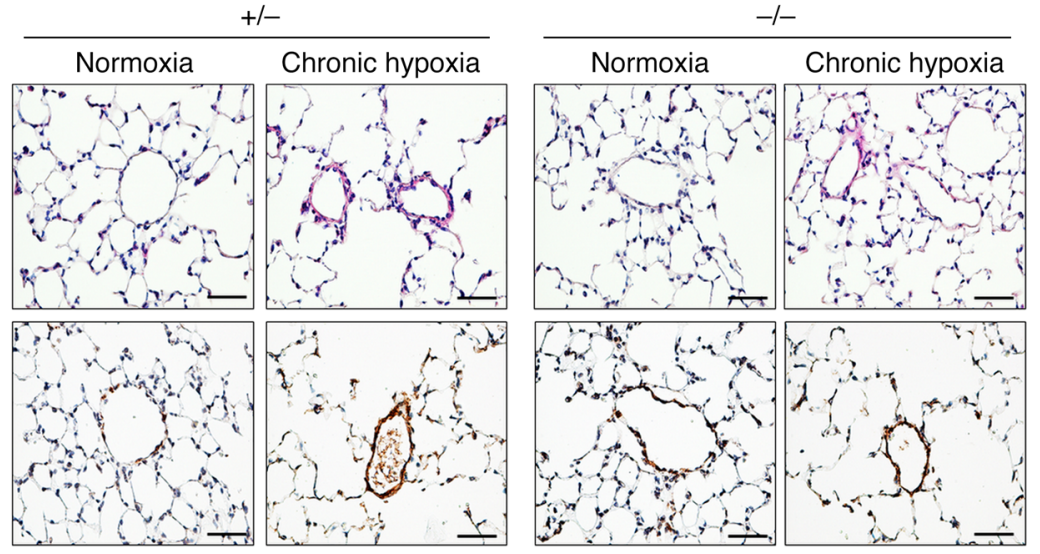

Figure 5. Susceptibility of transgenic mice deficient in IL6R in the smooth muscle (Sm22a-Cre II6ril/fl mice) to the development of chronic hypoxiainduced PH. (A) RVSP ( $n=6-11)$ and (B) RVH expressed by the Fulton index in Sm22a-Cre $/ / 6 r^{f l / f l}(-/-)$, Sm22a-Cre $/ / 6 r^{f l /+}(+/-)$, and Sm22a-Cre $/ / 6 r^{+/+}(+/+)$ mice $(n=8-11)$. (C) Representative images of H\&E and $\alpha$-SMA staining and quantification of the percentage of muscularized distal pulmonary arteries in lungs from Sm22a-Cre $\| 6 r^{f / f f l}$, Sm22a-Cre $\| 6 r^{f / /+}$, and Sm22a-Cre $/ / 6 r^{+/+}$mice $(n=6)$. Scale bars: $50 \mu \mathrm{m}$. Data are presented as mean \pm SEM. Comparisons were made using 2-way ANOVA with Bonferroni's post hoc tests. ${ }^{* *} P<0.001,{ }^{* * *} P<0.0001$ versus Sm22a-Cre $1 / 6 r^{+/+}$mice under normoxia; $P<0.05$, ${ }^{\# \#} P<0.01, \# \# \# P<0.0001$ versus Sm22a-Cre $/ / 6 r^{+/+}$mice under chronic hypoxia. LV, left ventricle; RV, right ventricle; S, septum.

(3-5); however, the exact reasons for these associations are unknown. Here, our results show for the first time to our knowledge ectopic upregulation of membrane-bound IL6R in the smooth muscle layer of remodeled vessels in human $\mathrm{IPAH}$ and experimental $\mathrm{PH}$. Furthermore, we demonstrate a key role for IL6R-dependent signaling in PA-SMC accumulation in vitro and in vivo. To specifically target IL6R in the smooth muscle layer, we used $S m 22 a-C r e ~ I l 6 r^{f l / f l}$ mice and revealed the potent pro-survival potential of this membrane-bound receptor. These transgenic animals displayed reduced PA-SMC accumulation and were protected against chronic hypoxia-induced $\mathrm{PH}$. Moreover, we determined that treatment with IL6R-specific antagonist prevent and/ or reverse experimental $\mathrm{PH}$ in 2 rat models. This therapeutic strategy holds promise for future clinical studies in $\mathrm{PAH}$.

Inflammatory processes are prominent in various types of human and experimental $\mathrm{PH}$ and are increasingly recognized as 
A
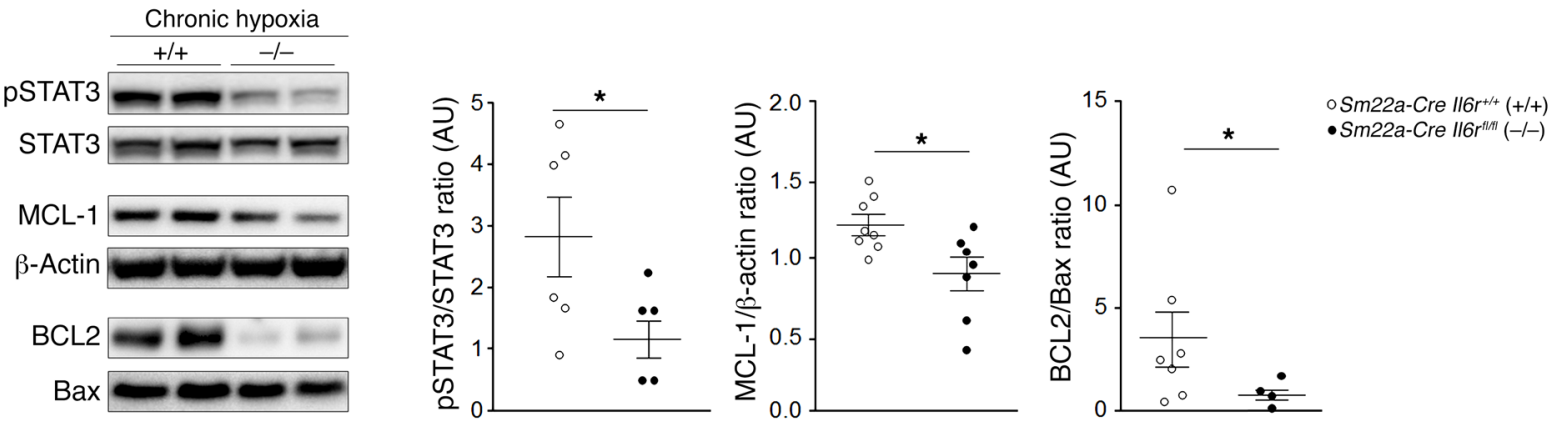

B
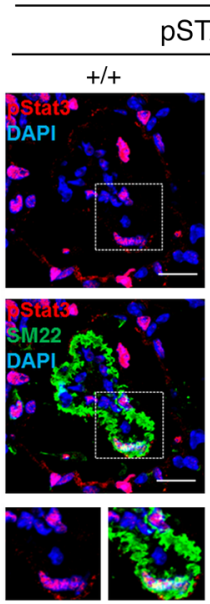

Chronic hypoxia

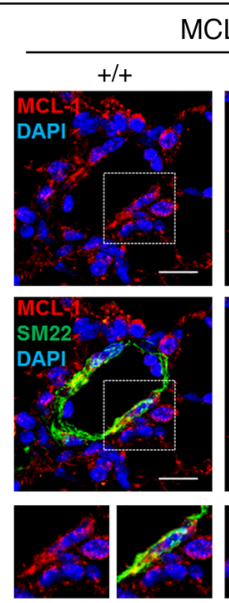

MCL-1

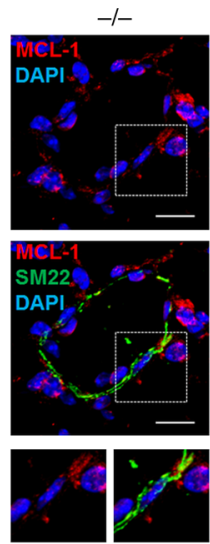

C

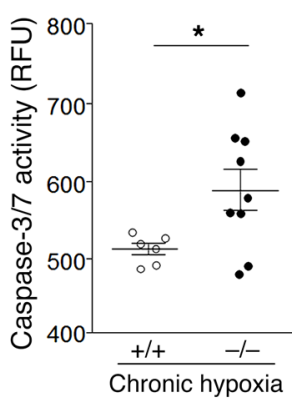

D

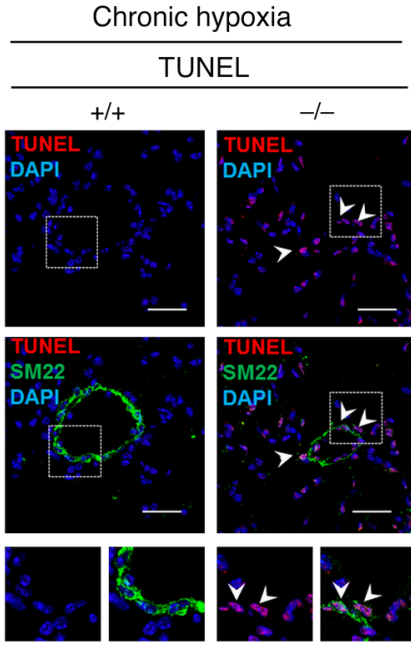

Figure 6. Reduced susceptibility of $S m 22 a-C r e ~ I I 6 r^{f l / f l}$ mice to the development of chronic hypoxia-induced PH is related to the downregulation of the antiapoptotic proteins MCL-1 and BCL2. (A) Representative Western blots and quantification of pSTAT3/STAT3, MCL-1/ $\beta$-actin, and BCL2/Bax ratios in lungs from Sm22a-Cre $/ / 6 r^{f / f f l}$ and Sm22a-Cre $\| 6 r^{+/+}$mice under chronic hypoxia $(n=4-8)$. (B) Double staining for pSTAT3 or MCL-1 with SM22 and DAPI in lungs from Sm22a-Cre $/ / 6 r^{f / / f l}$ and Sm22a-Cre $/ / 6 r^{+/+}$mice under chronic hypoxia $(n=4)$. (C) Levels of caspase-3/7 activity and (D) double staining with TUNEL, SM22, and DAPI in lungs from Sm22a-Cre II6r fl/fl and Sm22a-Cre $/ / 6 r^{+/+}$mice under chronic hypoxia ( $n=6-9$ ). Scale bars: $50 \mu \mathrm{m}$ (low magnification) and $70 \mu \mathrm{m}$ (high magnification). Arrowheads: positive cells. Data are presented as mean \pm SEM. Comparisons were made using the nonparametric Mann-Whitney $U$ test. ${ }^{*} P<0.05$ versus Sm22a-Cre $1 / 6 r^{+/+}$mice under chronic hypoxia.

major pathogenic components of pulmonary vascular remodeling. We recently found that dysfunctional pulmonary endothelium from patients with iPAH exhibits a steady-state proinflammatory phenotype in situ and in vitro and is a local source of IL-6 (21) together with resident M2 alveolar macrophages (20), opening doors to new therapeutical strategies in PAH.

The cellular distribution of IL-6 signaling differs substantially in the cis- and trans-mediated cascades. Indeed, cis-signaling relies on IL-6 binding the membrane IL6R, which in turn leads to dimerization of the second receptor subunit gp130 and initiation of intracellular signaling cascades that involved JAK/STAT. In contrast to gp130, which is ubiquitously in all cell types, IL6R expression is normally restricted to hepatocytes, neutrophils, monocytes/macrophages, and some lymphocytes. Alternatively, IL-6 can engage a soluble form of IL6R that is generated by RNA alternative splicing or, more frequently, by proteolytic cleavage of membrane IL6R mainly governed by a disintegrin and metalloproteinase 10 (ADAM10) (22). In this alternative IL-6 (trans) signaling pathway, sIL6R is responsible for chaperoning the cytokine and widening the spectrum of IL-6-responsive cells to those that lack membrane-bound IL6R at their cell surface. It is now well established that many inflammatory and neoplastic disorders are driven by IL-6 trans-signaling, which could lead to decreased endothelium-dependent vasorelaxation, generation of autoimmune Th17 cells, inhibition of T cell apoptosis, or monocyte differentiation or even facilitate cell accumulation through modulation of cell growth, proliferation, survival, and metabolism, among other effects. By studying the global expression pattern of key actors of IL-6 signaling in iPAH, we found an unexpected strong ectopic upregulation of membrane-bound IL6R in the smooth muscle layer of remodeled distal pulmonary arteries and cultured PA-SMCs derived from $\mathrm{PAH}$ patients together with high gp130 protein levels.

In order to obtain a better understanding of the functional role of the upregulation of membrane-bound IL6R in PA-SMCs, we performed a series of in vitro experiments with freshly isolated human PA-SMCs derived from lungs of patients with $\mathrm{PAH}$ and controls. These experiments reveal strong survival-promoting IL6R action in PA-SMCs in iPAH patients, as reflected by attenuation of caspase-3/7 activity levels induced by staurosporine, cycloheximide, and TNF- $\alpha$ as well as increased expression of the antiapoptotic proteins MCL-1 and BCL2 and high BCL2/Bax ratio when iPAH PA-SMCs were exposed to rHuIL-6. These observa- 

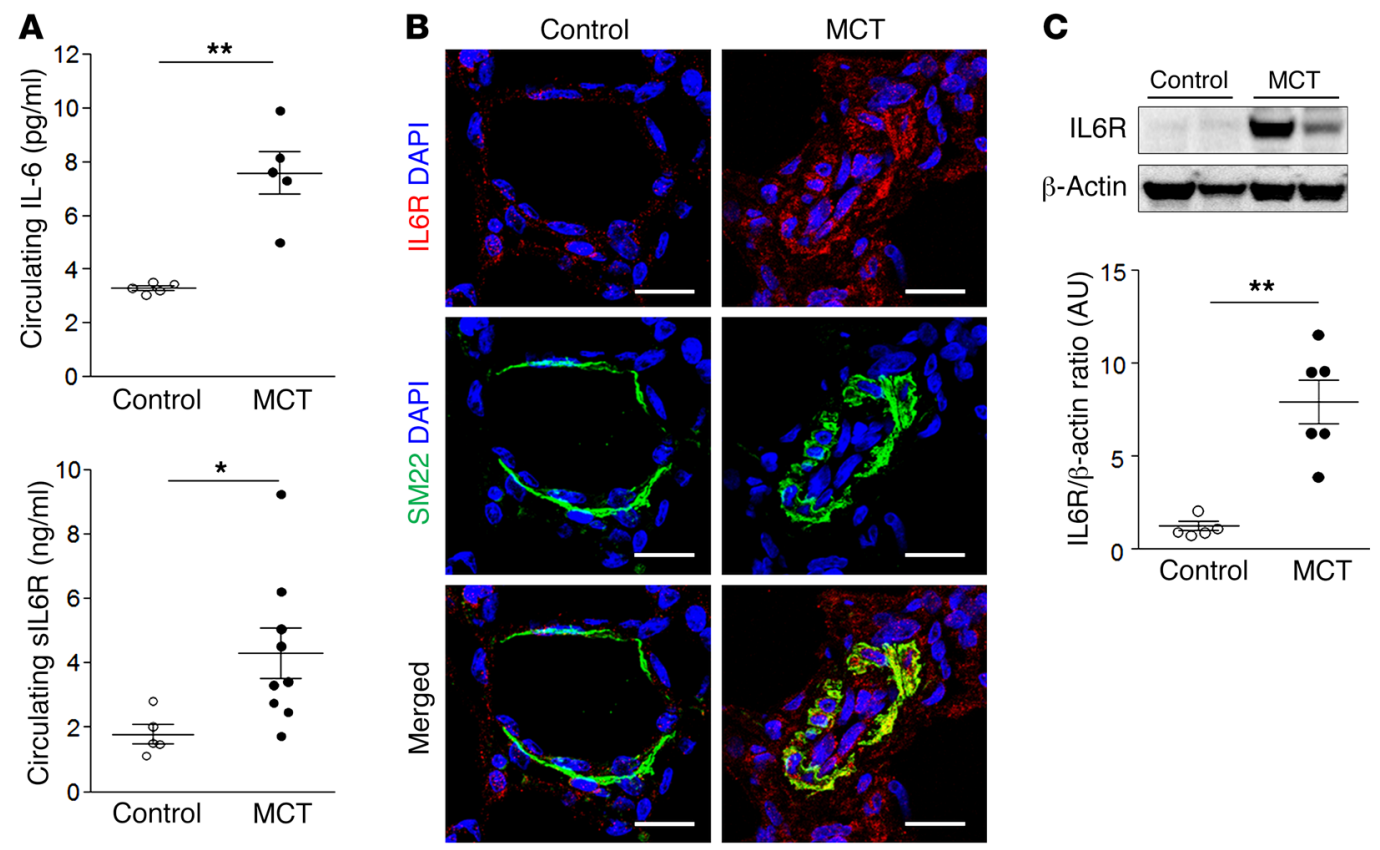

Figure 7. Increased circulating IL-6 and sIL6R levels and lung levels of membrane-bound IL6R in the MCT rat model of PH. (A) Circulating IL-6 and sIL6R levels $(n=5-9)$ and (B) double staining for IL6R and SM22 with DAPI in rat lungs in MCT-induced PH $(n=5)$. (C) Representative Western blots and quantification of the IL6R/ $\beta$-actin ratio in rat lungs in MCT-induced PH $(n=5-6)$. Scale bars: $50 \mu \mathrm{m}$. Data are presented as mean \pm SEM. Comparisons were made using the nonparametric Mann-Whitney $U$ test. ${ }^{*} P<0.05,{ }^{* *} P<0.01$ versus control rats.

tions were further validated by the overactivation of the IL-6/ IL6R axis in these cells and by the finding that both the neutralizing anti-IL6R and ERBF can significantly attenuate the apoptosisresistant PA-SMC phenotype in $\mathrm{PAH}$ and reduce the expression of key proapoptotic proteins in these cells. The pro-survival action of this ectopic IL6R upregulation could likely contribute to the phenotypic abnormalities observed in IPAH PA-SMCs that are characterized by an aberrant pro-proliferative and apoptosisresistant phenotype and by changes in metabolic processes. Although the precise mechanism underlying IL6R upregulation in PA-SMCs remains obscure, we found that in targeting this inherent intrinsic abnormality, we could partially restore their susceptibility to apoptosis in vitro and in vivo and therefore reduce the smooth muscle hyperplasia and progressive lumen narrowing in rodents in the most commonly used animal PH models, namely the chronic hypoxia, MCT, and SuHx models. Indeed, by exposing Sm22a-Cre $I l 6 r^{A / f l}$ mice, in which the Il6r gene is deleted in SM22-expressing cells, to chronic hypoxia or by treating rats daily with IL6R/sIL6R antagonist, we were able to prevent or reverse established $\mathrm{PH}$ in these complementary animal models.

A previous study by Brock et al. (23) demonstrated that IL-6 leads to repressed protein expression of bone morphogenetic protein receptor II (BMPRII) through overexpression of miR-17/92. Consistent with this finding, the inhibition of cis- and trans-mediated IL- 6 signaling with a gp130 inhibitor has been recently shown to upregulate BMPRII expression in the MCT rat model of $\mathrm{PH}$ (24). In the pathogenesis of PAH, dysfunctional BMPRII signaling is known to be involved in sustained inflammation and impaired resolution (25-28), although the underlying mechanism remains to be investigated (29). Furthermore, Davies et al. (30) have reported that PA-SMCs derived from patients carrying a BMPR2 mutation exhibited enhanced IL- 6 and IL- 8 induction by TGF- $\beta 1$, an effect that was reversed by NF-KB inhibition. Since crosstalk exists between BMP and TGF- $\beta$ signaling (31), further studies are needed to better understand the importance of ectopic upregulation of membrane-bound IL6R in this complex interplay. Such knowledge may help in drug management for patients with PAH. Indeed, administration of tocilizumab, a humanized anti-IL-6 monoclonal antibody approved for the treatment of rheumatoid arthritis in adults and children, has improved PAH symptoms in a patient with mixed connective tissue disease and severe PAH (32), and an open label study has been launched to assess the safety and efficacy of tocilizumab in group $1 \mathrm{PAH}$ patients (ClinicalTrials.gov, NCT02676947). Of note, it has been demonstrated that SNPs in the IL6R gene (e.g., Asp358Ala [rs2228145]; ref. 33) can influence inflammation in the context of various inflammatory (34-36) and cardiac disorders (37-39).

In summary, these findings offer insights into the role of the ectopic membrane-bound IL6R upregulation in PA-SMCs in PAH. Our data show that IL6R upregulation amplifies the survivalstimulating effect of IL- 6 through overexpression of the antiapoptotic proteins MCL-1 and BCL2 and contributes to excessive accumulation of PA-SMCs within the pulmonary arterial wall, ultimately leading to $\mathrm{PAH}$ progression. Interestingly, our in vivo experiments indicate that deletion of IL6R specifically in the smooth muscle layer prevents the development of experimental PH. Finally, we show here that in vivo IL6R inhibition reverses experimental $\mathrm{PH}$ in different rodent models of $\mathrm{PH}$. Our work suggests that IL6R blockade represents the best option within the IL-6/IL6R/gp130 signaling blockade, since this strategy spe- 
A Preventive protocol in the MCT model:

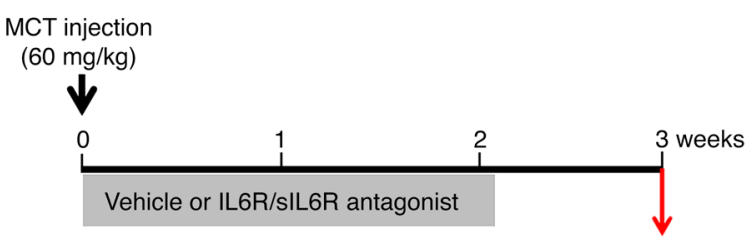

Sacrifice and

Hemodynamic measurements Histological analyses

\section{Curative protocol in the MCT model:}

MCT injection

(60 mg/kg)

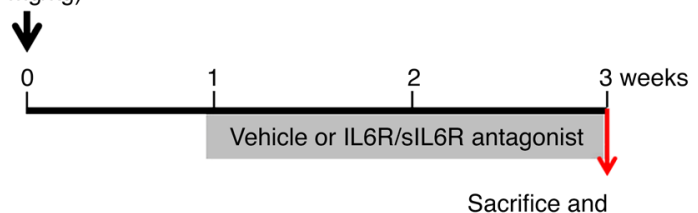

Hemodynamic meas Histological analyses

C

Preventive protoco in the MCT model:
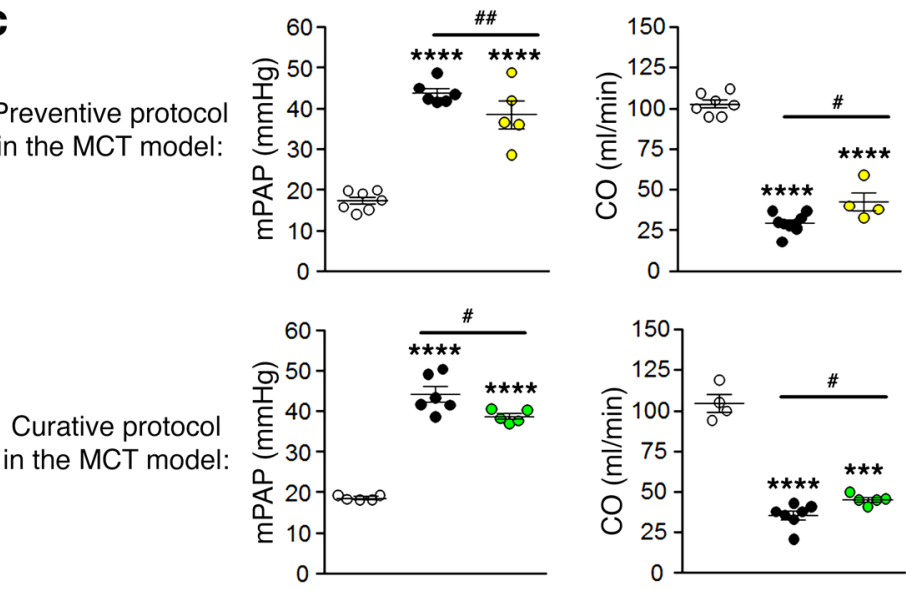

o Control

- MCT + vehicle

- MCT + IL6R/sIL6R antagonist (preventive protocol)

o MCT + IL6R/sIL6R antagonist (curative protocol)

B
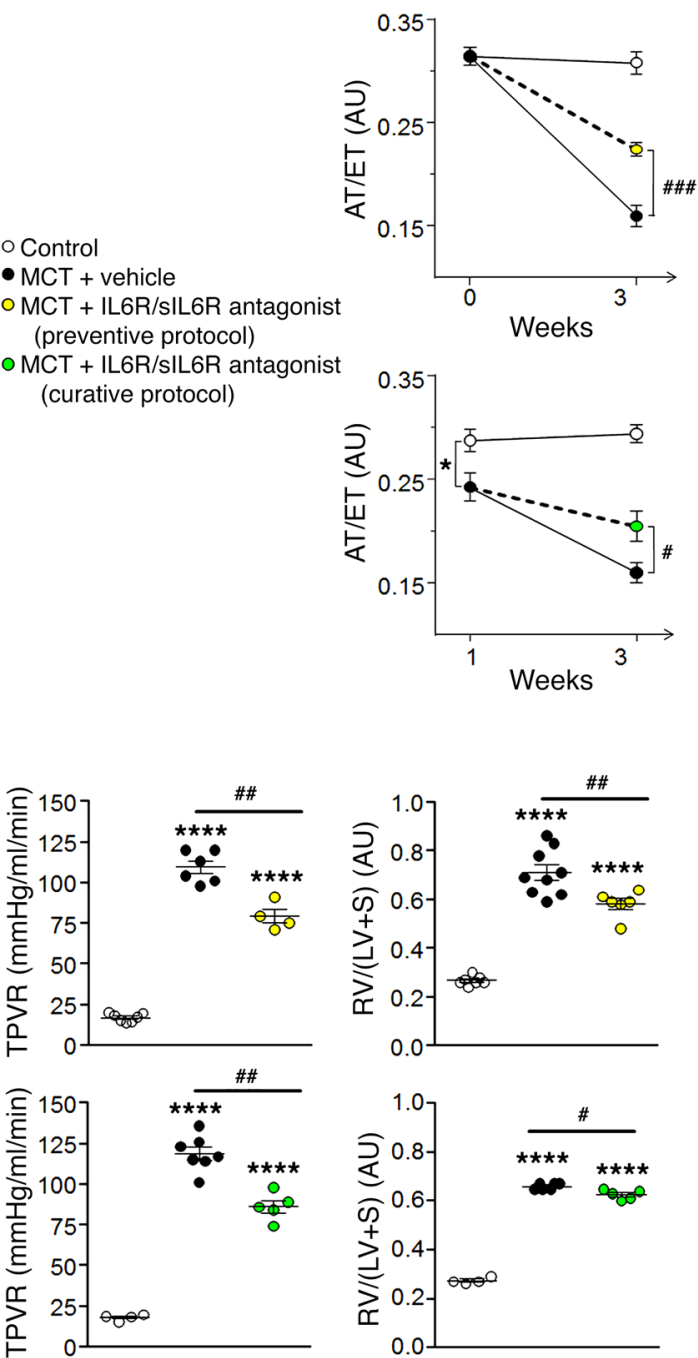
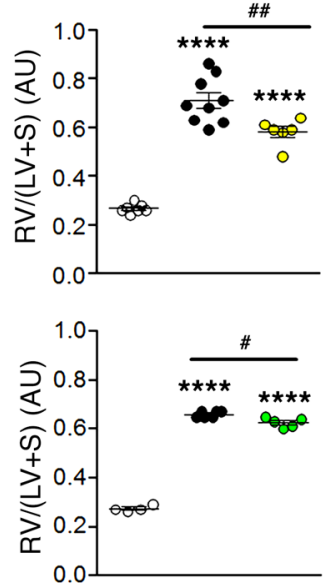

Figure 8. Efficacy of chronic treatment with the IL6R/sIL6R antagonist ERBF in the MCT rat model of PH. (A) Experimental strategies used to test the preventive and curative effects of IL6R/sIL6R antagonist in the MCT rat model of PH. (B) AT/ET ratios obtained by transthoracic echocardiography ( $n=$ 5-9). (C) mPAP, CO, TPVR, and right ventricular hypertrophy by the Fulton index $(n=4-9)$. Data are presented as mean \pm SEM. Comparisons were made using the nonparametric Mann-Whitney $U$ test or 2-way ANOVA with Bonferroni's post hoc tests. ${ }^{*} P<0.05,{ }^{* * *} P<0.001,{ }^{* * * *} P<0.0001$ versus control

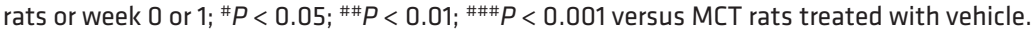

cifically targets PA-SMC proliferation and vascular remodeling. Specific IL6R blockade, and thereby classical signaling blockade (cis), could be therapeutically relevant for patients and holds promise for innovative PAH treatments.

\section{Methods}

Study population. Blood samples were taken from $\mathrm{PAH}$ and heritable PAH (hPAH) patients and normal subjects (Table 1). For the in vitro and in situ studies, we used lung specimens obtained during lung transplantation in IPAH patients and during lobectomy or pneumonectomy for localized lung cancer in control individuals. Preoperative echocardiological evaluation including echocardiography was performed in the controls to rule out PAH, and the lung specimens from the controls were collected at a distance from tumor foci.

Animals and in vivo treatment. To generate mice with smooth muscle cell-specific deletion of $\operatorname{Il6r}(\operatorname{Sm} 22 a$-Cre Il6r/f/ll mice), we crossed mice homozygous for the floxed IL6R allele (B6;SJL-Il6ratm1.1Drew/J, The Jackson Laboratory) with Sm22a-Cre mice (B6.129S6-Taglntm2 (cre) Yec/J, provided by S. Cohen-Kaminsky (INSERM UMR_S 999, Le Plessis-Robinson, France). The offspring were intercrossed. The offspring genotypes were determined by PCR as recommended by the Jackson Laboratory, and we confirmed that the mice are viable and fertile, and have a normal life span and growth rate. The resulted offspring, Sm22a-Cre Il $6 r^{a / / l}$ mice and their littermates, $\mathrm{Sm} 22 \mathrm{a}$-Cre Il6r+/+ mice, were used for all experiments. The mice were either studied in room air at 8 weeks of age or were exposed to hypoxia $\left(\mathrm{FiO}_{2}, 10 \%\right)$ for 3 weeks. As previously described $(40,41)$, hemodynamic parameters were measured in unventilated anesthetized mice (isoflurane) using a closed chest technique, by introducing a 1.4-F Millar catheter (ADInstruments) into the jugular vein and directing it to the right ventricle to assess RVSP. RVH was calculated using the Fulton index [weight ratio of right ventricle and (left ventricle + septum)]. 
A

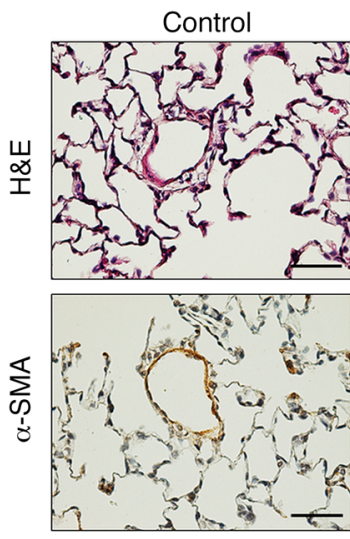

MCT + IL6R/sIL6R antagonist
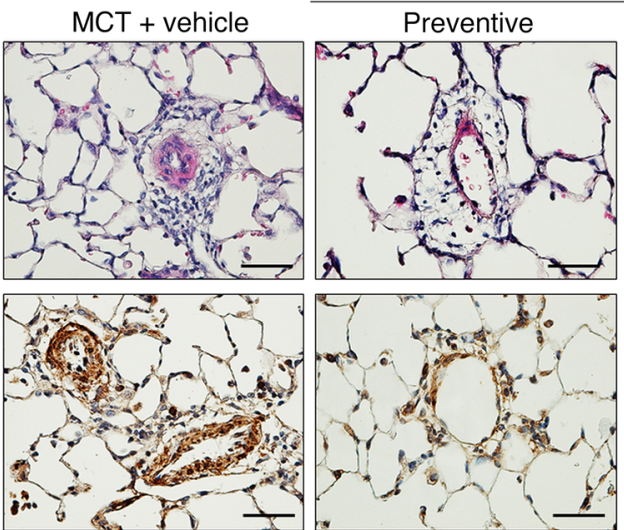

Preventive
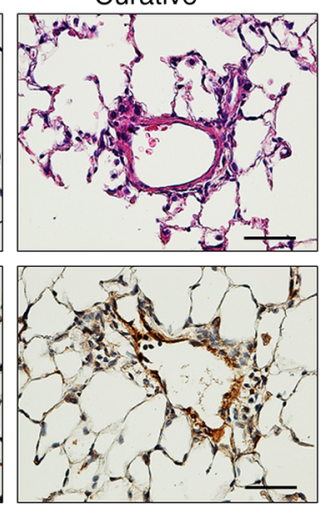

Control

- MCT + vehicle

OMCT + IL6R/sIL6R antagonist (preventive protocol)

OMCT + IL6R/sIL6R antagonist (curative protocol)
B

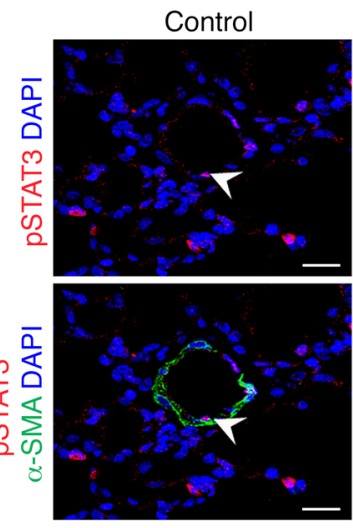

C

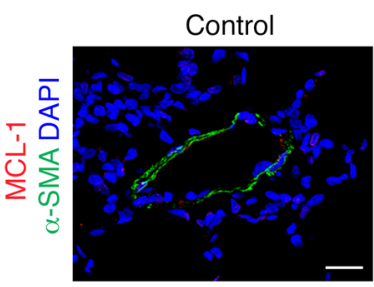

D

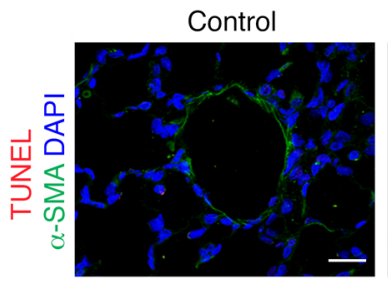

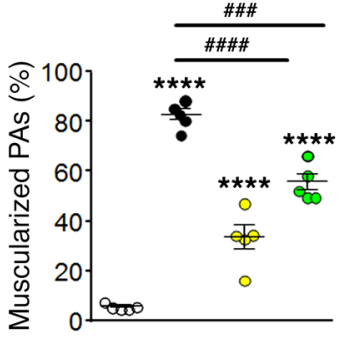

MCT + IL6R/sIL6R antagonist
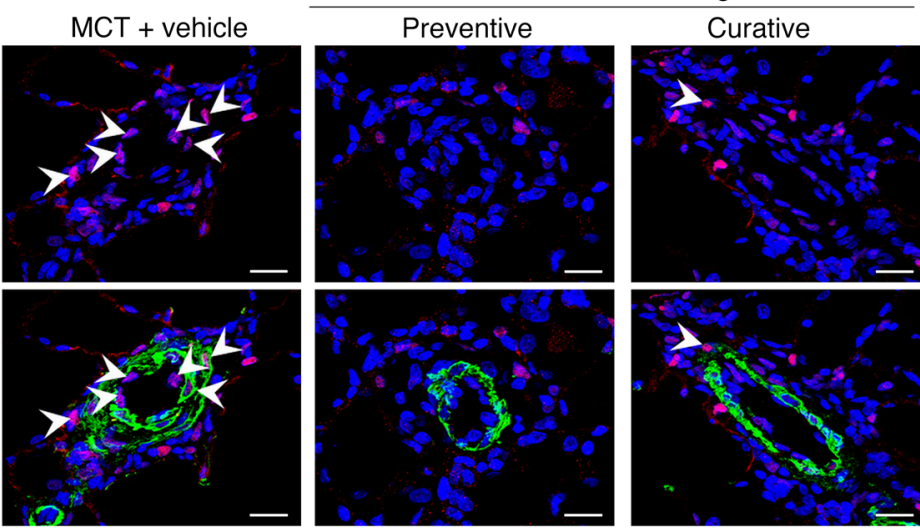

MCT + IL6R/sIL6R antagonist
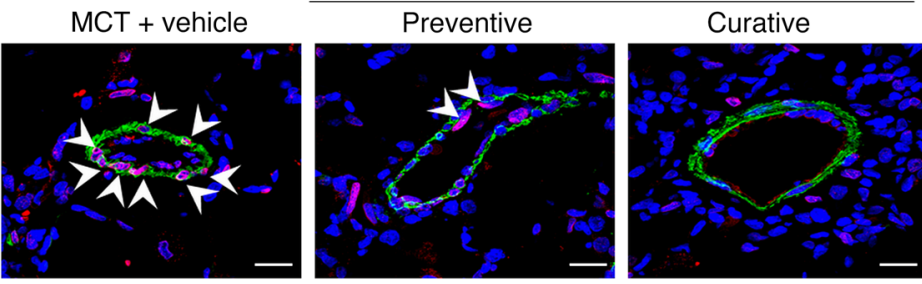

MCT + IL6R/sIL6R antagonist

$\mathrm{MCT}$ + vehicle

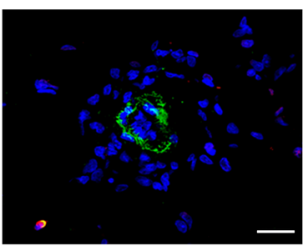

Preventive

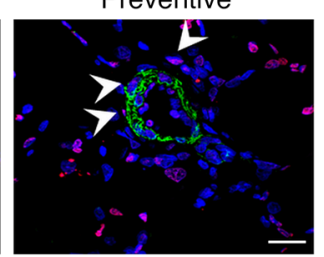

Curative

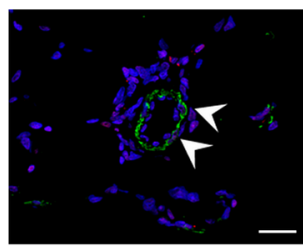

Figure 9. Chronic treatment with the IL6R/sIL6R antagonist ERBF attenuates pulmonary vascular remodeling in MCT-injected rats. (A) Representative images of H\&E and $\alpha$-SMA immunostaining, and quantification of the percentage of wall thickness and of muscularized distal pulmonary arteries (PAs) in lungs of MCT rats treated or not with IL6R/sIL6R antagonist $(n=$ 5). (B) Double staining for pSTAT3 and $\alpha$-SMA with DAPI in lungs of MCT rats treated or not with IL6R/ slL6R antagonist $(n=5)$. (C) Double staining for MCL-1 or (D) TUNEL with $\alpha$-SMA and DAPI in lungs of MCT rats treated or not with IL6R/ sIL6R antagonist $(n=5)$. Scale bars: $50 \mu \mathrm{m}$. Arrowheads: positive cells. Data are presented as mean \pm SEM. Comparisons were made using 2-way ANOVA with Bonferroni's post hoc tests. ${ }^{*} P<0.05$, ${ }^{* *} P<0.01$, ${ }^{* * *} P<0.0001$ versus control rats; ${ }^{\# \# P}<0.001$, \#\#\#\# $P$ $<0.0001$ versus MCT rats treated with vehicle. 
A
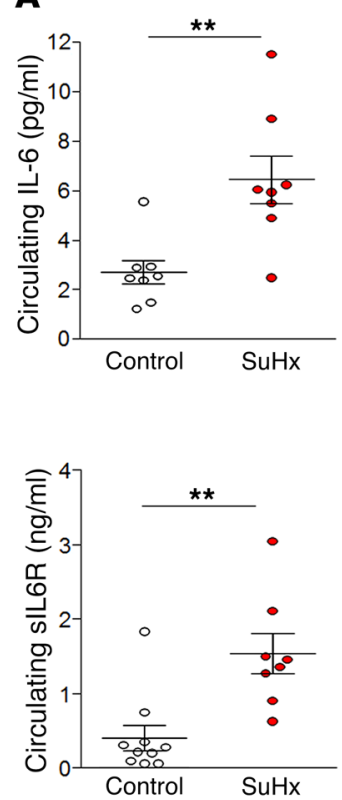

B
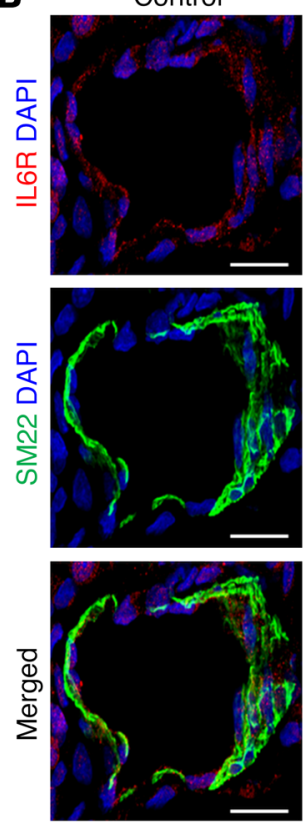

SuHx
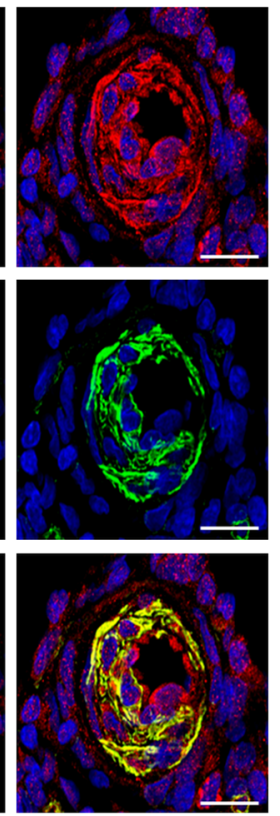

C
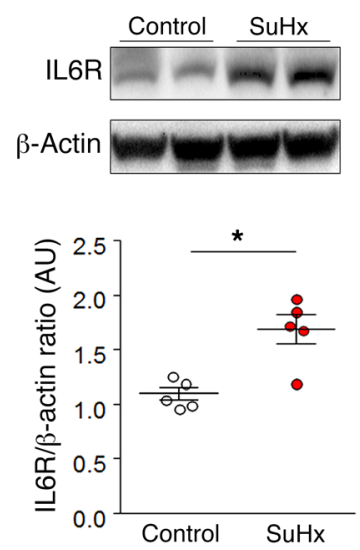

D

Curative protocol in the SuHx model:

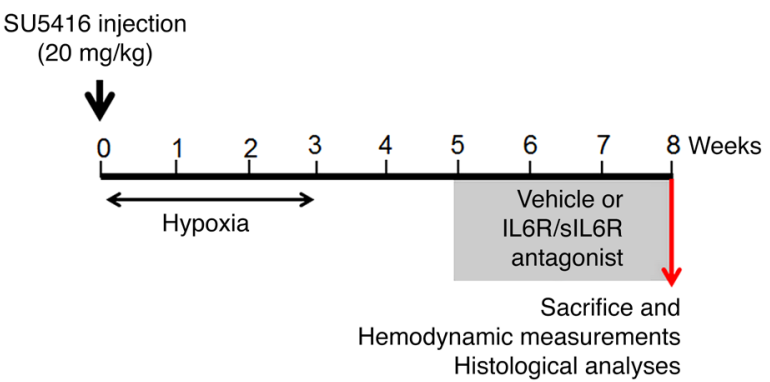

Histological analyses

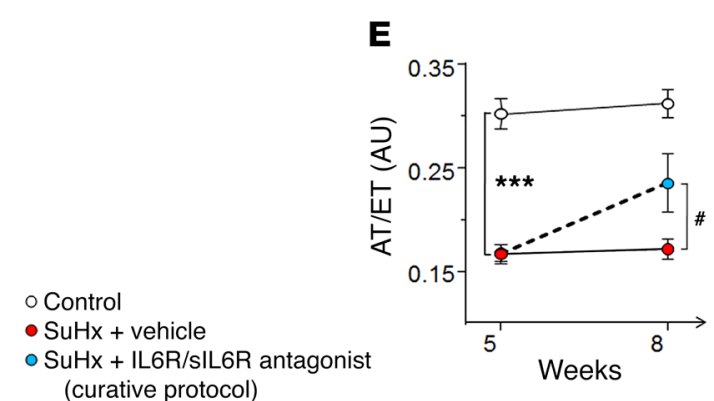

(curative protocol)
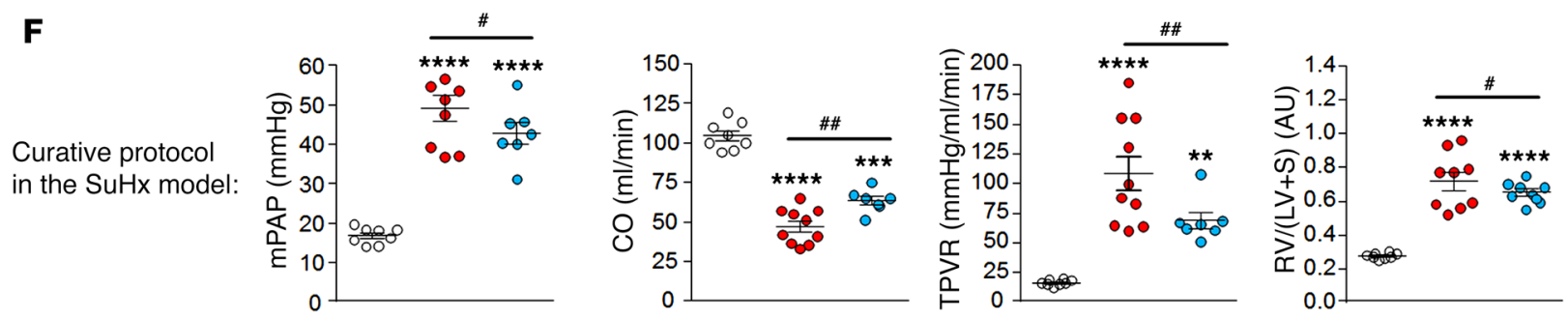

Figure 10. Efficacy of chronic treatment with the IL6R/sIL6R antagonist ERBF in the SuHx rat model of severe PH. (A) Circulating IL-6 and sIL6R levels $(n=8-10)$ and (B) double staining for IL6R and SM22 with DAPI in rat lungs in SuHx-induced PH $(n=4)$. (C) Representative Western blots and quantification of the IL6R/ $\beta$-actin ratio in rat lungs in SuHx-induced PH $(n=5)$. (D) Experimental strategies used to test the curative effect of IL6R/sIL6R antagonist in the SuHx rat model of severe PH. (E) AT/ET ratios obtained by transthoracic echocardiography $(n=6-10)$. (F) mPAP, CO, TPVR and RVH by the Fulton index $(n=6-10)$. Scale bars: $50 \mu \mathrm{m}$. Data are presented as mean \pm SEM. Comparisons were made using the nonparametric Mann-Whitney $U$ test or 2 -way

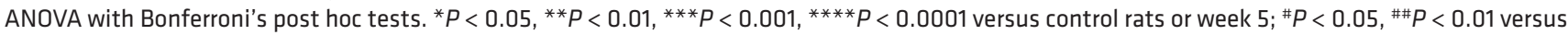
SuHx rats treated with vehicle.

Young male Wistar rats (100 g, Janvier Labs) were subjected to 2 different PH models: a single $60 \mathrm{mg} / \mathrm{kg}$ dose of subcutaneously injected MCT (Sigma-Aldrich) or a single $20-\mathrm{mg} / \mathrm{kg}$ dose of subcutaneously injected SU5416 (SUGEN, Mifcare), followed by 3 weeks of chronic hypoxia $\left(\mathrm{FiO}_{2}, 10 \%\right)$ and 5 weeks of normoxia. A subgroup of rats was treated daily with i.p. injection of IL6R/sIL6R antagonist
(Mifcare) at a dose of $0.5 \mathrm{mg} / \mathrm{kg} / \mathrm{d}$ (17) for 2 or 3 weeks according to experimental strategies. Pulsed-wave Doppler during transthoracic echocardiography was used to evaluate the ratio of pulmonary artery acceleration time (AT) to right ventricular ejection time (ET), before and after IL6R/sIL6R antagonist treatments, using Vivid E9 (GE Healthcare). At the end of these protocols, as previously described 
A
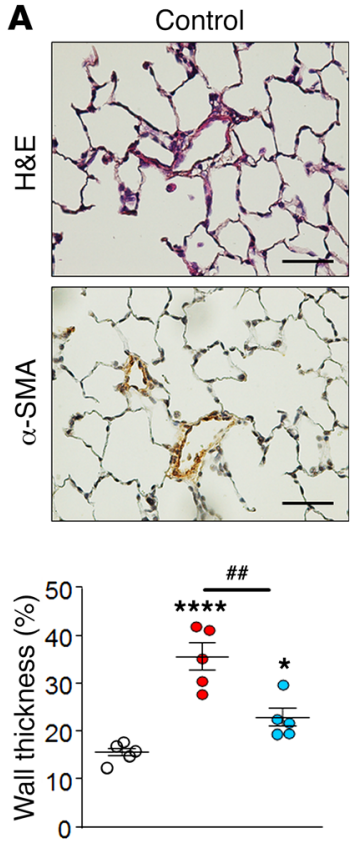

B
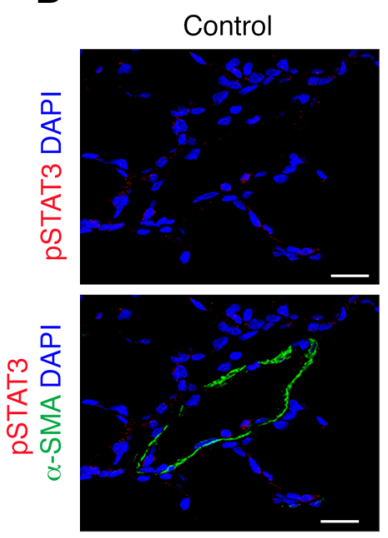

C

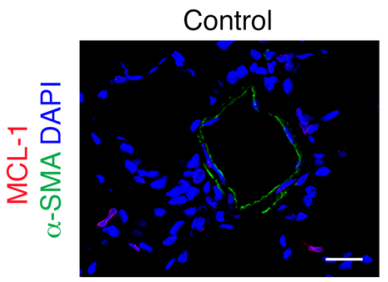

D

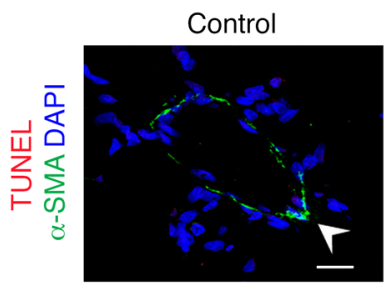

$\mathrm{SuHx}+$
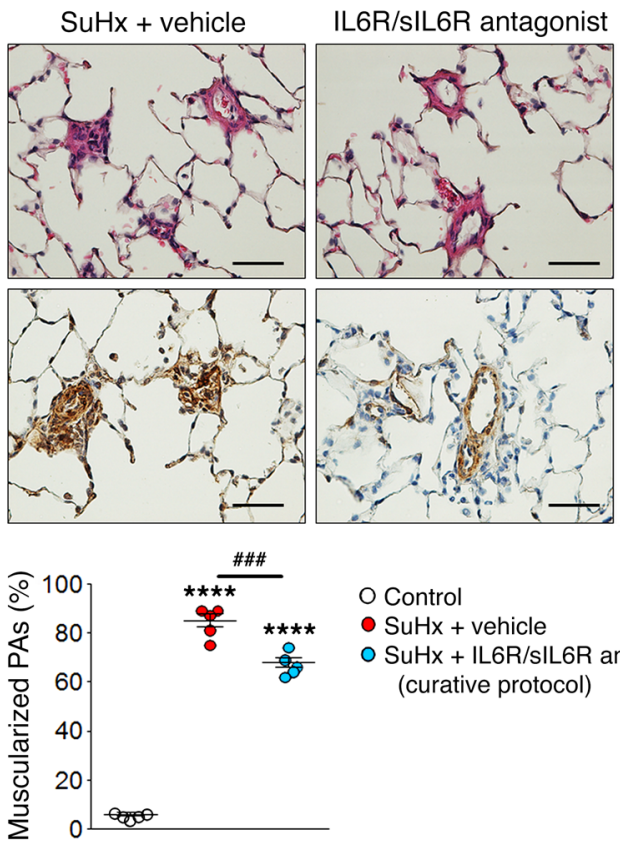

O Control

- SuHx + vehicle

o SuHx + IL6R/sIL6R antagonist (curative protocol)

$\mathrm{SuHx}+$

SuHx + vehicle
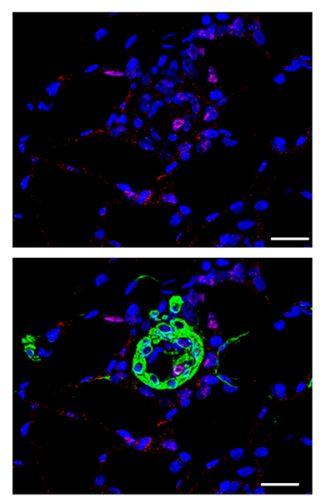

SuHx + vehicle

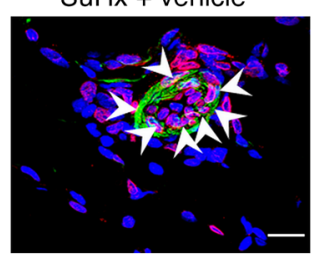

SuHx + vehicle

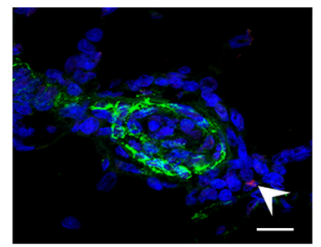

IL6R/sIL6R antagonist
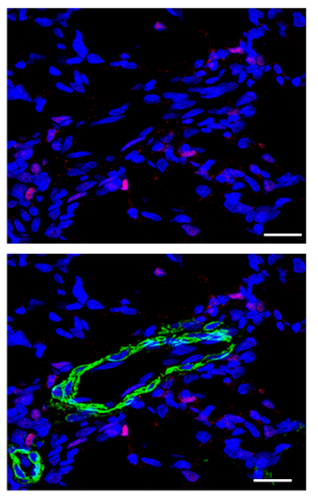

$\mathrm{SuHx}+$

IL6R/sIL6R antagonist

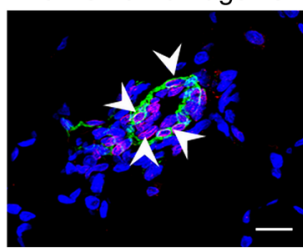

$\mathrm{SuHx}+$

IL6R/sIL6R antagonist

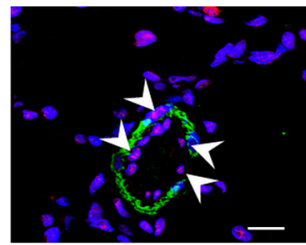

Figure 11. Chronic treatment with the IL6R/sIL6R antagonist ERBF attenuates pulmonary vascular remodeling in SuHx rats. (A) Representative images of $H \& E$ and $\alpha$-SMA immunostaining, and quantification of the percentage of wall thickness and of muscularized distal pulmonary arteries in lungs of SuHx rats treated or not with IL6R/sIL6R antagonist $(n=5)$. (B) Double staining for $\mathrm{PSTAT} 3$ and $\alpha$-SMA with DAPI in lungs of SuHx rats treated or not with IL6R/sIL6R antagonist $(n=5)$. (C) Double staining for MCL-1 or (D) TUNEL with $\alpha$-SMA and DAPI in lungs of SuHx rats treated or not with IL6R/sIL6R antagonist $(n=5)$. Scale bars: $50 \mu \mathrm{m}$. Arrowheads: positive cells. Data are presented as mean \pm SEM. Comparisons were made using 2-way ANOVA with Bonferroni's post hoc tests. ${ }^{*} P<0.05$, ${ }^{* * *} P<$

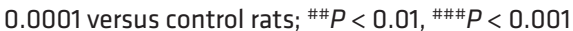
versus SuHx rats treated with vehicle.

ment of hemodynamic parameters, the thorax was opened and the left lung immediately removed and frozen. The right lung was fixed in the distended state with formalin buffer. RVH was assessed by the Fulton index, and the percentages of wall thickness [ $(2 \times$ medial wall thickness/external diameter $)$ $\times 100]$ and muscularized vessels were determined. To assess pulmonary vasoconstriction in response to acute hypoxia, we determined values of MPAP in vehicle- and ERBF-treated healthy rats ventilated with room air (baseline) and after 10 minutes of ventilation with a hypoxic gas mixture $(41,45,46)$.

Isolation and culture of murine and human PA-SMCs. Primary human PA-SMCs were isolated from lung explants from $\mathrm{PAH}$ patients or from lung specimens obtained during lobectomy or pneumonectomy at a distance from tumor foci in control subjects. Preoperative echocardiography was performed in these control patients to rule out $\mathrm{PH}$, and the absence of tumoral infiltration was retrospectively established in all tissue sections by histopathological analysis. Briefly, as previously described (14, $42-44,47,48)$, small pieces of freshly isolated arteries were cultured in DMEM medium supplemented with 15\% FCS, 2 mM L-glutamine, and antibiotics. The isolated pulmonary PA-SMCs were strongly positive for $\alpha$-smooth muscle actin ( $\alpha$-SMA), smooth muscle-specific SM22 protein, and calponin and negative for von Willebrand factor and CD31. Cells were used at passages less than 5. For overexpression of IL6R, PA-SMCs were transfected using Lipofectamine 2000 with $1.5 \mu \mathrm{g}$ of a full-length human IL6R ( $p C M V 3-I L 6 R$-GFPSpark, Interchim) construct in pCMV3-C-GFPSpark or with $1.5 \mu \mathrm{g}$ of $p C M V 3-C$ GFPSpark control plasmid ( $p C M V 3-I L 6 R$-GFPSpark, Interchim). The cells were studied within 3 days after transfection. Overexpression of IL6R levels was doc-

$(14,21,42-44)$, animals were anesthetized with isoflurane. A polyvinyl catheter was introduced into the right jugular vein and pushed through the right ventricle into the pulmonary artery to measure MPAP. CO in rats was measured using the thermodilution method. After measure- umented 72 hours after transfection.

Primary murine PA-SMCs were isolated from Sm22a-Cre Il6r $r^{f / f l}$ and Sm22a-Cre $\mathrm{Il}^{+/+}$mice using a modified elastase/collagenase digestion protocol. Briefly, after anesthesia, central pulmonary arter- 
ies were carefully dissected in DMEM medium containing $40 \mu \mathrm{mol} / 1$ $\mathrm{CaCl}_{2}$, cut into $<1-\mathrm{mm}^{3}$ pieces, and digested with collagenase $(1 \mathrm{mg} / \mathrm{ml})$ and elastase $(1 \mathrm{mg} / \mathrm{ml})$ for 20 minutes at $37^{\circ} \mathrm{C}$. Then PA-SMCs were collected through centrifugation at $300 \mathrm{~g}$ for 10 minutes and subcultured with DMEM containing 10\% FCS and antibiotics. PA-SMCs were characterized by being positive for $\alpha$-SMA ( $>95 \%)$ and negative for endothelial cell marker CD31 by immunostaining.

Western blot analysis, ELISA, and immunostaining. Cells/tissues were homogenized and sonicated in RIPA buffer containing protease and phosphatase inhibitors and $30 \mu \mathrm{g}$ of protein was used to detect IL6R, gp130, pSTAT3, STAT3, Bax, BCL2, MCL-1 and $\beta$-actin (Supplemental Figure 4) (14, 42-44, 47, 48). Concentrations of IL-6, sIL6R, and sgp130 in human and rat serum were evaluated using a specific ELISA kit (R\&D Systems; Interchim) according to the manufacturer's instructions. Immunohistochemistry and immunocytofluorescence staining for IL6R, gp130, pSTAT3, SM22, CD45, CD68, F4/80, and $\alpha$-SMA (Supplemental Figure 4) were performed in human and rat lung paraffin sections $(14,21,42-44)$. Details on the sources of proteins are shown in Supplemental Table 1. Briefly, lung sections (5- $\mu \mathrm{m}$ thickness) were deparaffinized and stained with $\mathrm{H} \& \mathrm{E}$ (Sigma-Aldrich), Sirius red, or Masson's trichrome, or incubated with retrieval buffer. Then sections were saturated with blocking buffer and incubated overnight with specific antibodies, followed by corresponding secondary fluorescent-labeled antibodies (Thermo Fisher Scientific). Nuclei were labeled using DAPI (Thermo Fisher Scientific). Mounting was done using ProLong Gold antifade reagent (Thermo Fisher Scientific). Images were taken using an LSM700 confocal microscope (Zeiss). Other lung sections were used for immunochemistry using a VECTASTAIN ABC kit according to the manufacturer's instructions (Vector Laboratories) and counterstained with hematoxylin (Sigma-Aldrich). Images were taken using an Eclipse 80i microscope (Nikon Instruments).

Induction and detection of cell apoptosis and proliferation. To measure activity of caspase-3/7 using an Apo-ONE kit (Promega), we seeded cells in a 96-well plate $(46,49)$. After treatment with rHuIL-6 $(10 \mathrm{ng} / \mathrm{ml}$ ) (14) for 48 hours in medium containing 0.1\% BSA, the specific reagents (staurosporine, $0.1 \mu \mathrm{M}$, refs. 50, 51; cycloheximide, $50 \mu \mathrm{g} / \mathrm{ml}$, ref. 44 ; and TNF- $\alpha, 10 \mathrm{ng} / \mathrm{ml}$, ref. 52$)$ were added in each well and incubated for 2.5 hours. For anti-IL6R $(0.5 \mu \mathrm{g} / \mathrm{ml})$ and IL6R/ sIL6R antagonist $(10 \mu \mathrm{M})$ treatments (Supplemental Figure 1, C and D), cells were pretreated for 1 hour before being exposed to rHuIL- 6 (10 ng/ml). Fluorescence was monitored by EnVision (PerkinElmer). In addition, we conducted TUNEL staining using an ApopTag Red In Situ Apoptosis Detection Kit (Chemicon) according to the manufacturer's instructions. The number of TUNEL-positive nuclei per total number of nuclei was determined (46). Cell proliferation was assessed by BrdU using a DELFIA Cell Proliferation kit (PerkinElmer) (42).

Statistics. Statistical significance was tested using the nonparametric Mann-Whitney $U$ test or 2-way ANOVA with Bonferroni's post hoc tests. Continuous data are expressed as mean \pm SEM of at least 3 independent experiments performed in triplicate or quintuplicate for technical replicates. $P<0.05$ was considered statistically significant. Analyses were performed using GraphPad Prism version 5.0.

Study approval. All animals were treated in accordance with the NIH Guide for the Care and Use of Laboratory Animals as adopted by the French National Institute of Health and Medical Research (INSERM), and approval was granted by the Ethics Committee of University Paris-Sud, Le Plessis-Robinson, France. In addition, all experiments with human specimens were approved by the local ethics committee (Comité de Protection des Personnes [CPP] Ile-de-France VII). All patients gave written informed consent before the study.

\section{Author contributions}

YT, CP, LT, and CG conceptualized and designed the study; all authors analyzed and interpreted the data; CP, LT, AH, MH, and CG drafted the manuscript.

\section{Acknowledgments}

The authors thank Amélie Cumont and Jennifer Bordenave for their technical assistance. This research was supported by grants from INSERM, University Paris-Sud and the University ParisSaclay, Marie Lannelongue Hospital, Chancellerie des Universités de Paris (Legs Poix), French National Agency for Research (ANR) grant ANR-16-CE17-0014 (TAMIRAH), Fondation de la Recherche Médicale (FRM) grant DEQ20150331712 (Equipe FRM 2015), and National Agency for Drug Safety (ANSM) grant VIGIAPATH, and in part by Département Hospitalo-Universitaire (DHU) Thorax Innovation (TORINO); AP-HP; Service de Pneumologie, Centre de Référence de l'Hypertension Pulmonaire Sévère; LabEx LERMIT (grant ANR-10-LABX-0033); the French PAH patient association (HTAP France); and the French Fonds de Dotation "Recherche en Santé Respiratoire"-Fondation du Souffle (FRSRFdS). YT (RESPIRE2-2013-4919) is the recipient of a European Respiratory Society/European Union RESPIRE2 Marie Curie research fellowship. CP is supported by the FRSR-FdS.

Address correspondence to: Christophe Guignabert, INSERM UMR_S 999; 133, Avenue de la Résistance; 92350 Le PlessisRobinson, France. Phone: 33.1.40948833; Email: christophe. guignabert@inserm.fr.
1. Humbert M, Lau EM, Montani D, Jais X, Sitbon O, Simonneau G. Advances in therapeutic interventions for patients with pulmonary arterial hypertension. Circulation. 2014;130(24):2189-2208.

2. Boucly A, et al. Risk assessment, prognosis and guideline implementation in pulmonary arterial hypertension. Eur Respir J. 2017;50(2):1700889.

3. Cracowski JL, et al. Proinflammatory cytokine levels are linked to death in pulmonary arterial hypertension. Eur Respir J. 2014;43(3):915-917.

4. Matura LA, et al. Interleukin-6 and tumor necrosis factor- $\alpha$ are associated with quality of life- related symptoms in pulmonary arterial hypertension. Ann Am Thorac Soc. 2015;12(3):370-375.

5. Soon E, et al. Elevated levels of inflammatory cytokines predict survival in idiopathic and familial pulmonary arterial hypertension. Circulation. 2010;122(9):920-927.

6. Hunter CA, Jones SA. IL-6 as a keystone cytokine in health and disease. Nat Immunol. 2015;16(5):448-457.

7. Grivennikov SI, Karin M. Inflammatory cytokines in cancer: tumour necrosis factor and interleukin 6 take the stage. Ann Rheum Dis. 2011;
70(Suppl 1):i104-i108.

8. Rider P, Carmi Y, Cohen I. Biologics for targeting inflammatory cytokines, clinical uses, and limitations. Int J Cell Biol. 2016;2016:9259646.

9. Golembeski SM, West J, Tada Y, Fagan KA. Interleukin-6 causes mild pulmonary hypertension and augments hypoxia-induced pulmonary hypertension in mice. Chest. 2005; 128(6 Suppl):572S-573S.

10. Miyata M, Sakuma F, Yoshimura A, Ishikawa H, Nishimaki T, Kasukawa R. Pulmonary hypertension in rats. 2. Role of interleukin-6. 
International archives of allergy and immunology. 1995;108(3):287-291.

11. Savale $L$, et al. Impact of interleukin- 6 on hypoxiainduced pulmonary hypertension and lung inflammation in mice. Respir Res. 2009;10:6.

12. Steiner MK, Syrkina OL, Kolliputi N, Mark EJ, Hales CA, Waxman AB. Interleukin-6 overexpression induces pulmonary hypertension. Circ Res. 2009;104(2):236-244.

13. Humbert $\mathrm{M}$, et al. Increased interleukin-1 and interleukin-6 serum concentrations in severe primary pulmonary hypertension. Am J Respir Crit Care Med.1995;151(5):1628-1631.

14. Ricard N, et al. Increased pericyte coverage mediated by endothelial-derived fibroblast growth factor- 2 and interleukin- 6 is a source of smooth muscle-like cells in pulmonary hypertension. Circulation. 2014;129(15):1586-1597.

15. Courboulin A, et al. Krüppel-like factor 5 contributes to pulmonary artery smooth muscle proliferation and resistance to apoptosis in human pulmonary arterial hypertension. Respir Res. 2011;12:128.

16. Scheller J, Chalaris A, Schmidt-Arras D, RoseJohn S. The pro- and anti-inflammatory properties of the cytokine interleukin-6. Biochim Biophys Acta. 2011;1813(5):878-888.

17. Vardanyan M, et al. Reversal of pancreatitisinduced pain by an orally available, small molecule interleukin- 6 receptor antagonist. Pain. 2010;151(2):257-265.

18. Hayashi M, et al. Biological activity of a novel nonpeptide antagonist to the interleukin- 6 receptor 20S,21-epoxy-resibufogenin-3-formate. JPharmacol Exp Ther. 2002;303(1):104-109.

19. Kino T, et al. 3-O-Formyl-20R,21-epoxyresibufogenin suppresses IL-6-type cytokine actions by targeting the glycoprotein 130 subunit: potential clinical implications. JAllergy Clin Immunol. 2007;120(2):437-444.

20. Hashimoto-Kataoka T, et al. Interleukin-6/interleukin-21 signaling axis is critical in the pathogenesis of pulmonary arterial hypertension. Proc Natl Acad Sci U S A. 2015;112(20):E2677-E2686.

21. Le Hiress $M$, et al. Proinflammatory signature of the dysfunctional endothelium in pulmonary hypertension. role of the macrophage migration inhibitory factor/CD74 complex. Am J Respir Crit Care Med. 2015;192(8):983-997.

22. Schumacher $\mathrm{N}$, et al. Shedding of endogenous interleukin-6 receptor (IL-6R) is governed by a disintegrin and metalloproteinase (ADAM) proteases while a full-length IL-6R isoform localizes to circulating microvesicles. J Biol Chem. 2015;290(43):26059-26071.

23. Brock M, et al. Interleukin- 6 modulates the expression of the bone morphogenic protein receptor type II through a novel STAT3microRNA cluster 17/92 pathway. Circ Res. 2009;104(10):1184-1191.
24. Huang Z, et al. Glycoprotein 130 inhibitor ameliorates monocrotaline-induced pulmonary hypertension in rats. Can J Cardiol. 2016;32(11):1356.e1-1356.e10.

25. Sawada H, et al. Reduced BMPR2 expression induces GM-CSF translation and macrophage recruitment in humans and mice to exacerbate pulmonary hypertension. J Exp Med. 2014;211(2):263-280.

26. Song Y, Jones JE, Beppu H, Keaney JF Jr, Loscalzo J, Zhang YY. Increased susceptibility to pulmonary hypertension in heterozygous BMPR2mutant mice. Circulation. 2005;112(4):553-562.

27. Hong KH, et al. Genetic ablation of the BMPR2 gene in pulmonary endothelium is sufficient to predispose to pulmonary arterial hypertension. Circulation. 2008;118(7):722-730.

28. Hagen M, et al. Interaction of interleukin-6 and the BMP pathway in pulmonary smooth muscle. Am J Physiol Lung Cell Mol Physiol. 2007;292(6):L1473-L1479.

29. Guignabert C, Bailly S, Humbert M. Restoring BMPRII functions in pulmonary arterial hypertension: opportunities, challenges and limitations Expert Opin Ther Targets. 2017;21(2):181-190.

30. Davies RJ, et al. BMP type II receptor deficiency confers resistance to growth inhibition by TGF- $\beta$ in pulmonary artery smooth muscle cells: role of proinflammatory cytokines. Am J Physiol Lung Cell Mol Physiol. 2012;302(6):L604-L615.

31. Nasim MT, et al. BMPR-II deficiency elicits pro-proliferative and anti-apoptotic responses through the activation of TGF $\beta$-TAK1MAPK pathways in PAH. Hum Mol Genet. 2012;21(11):2548-2558.

32. Furuya $Y$, Satoh T, Kuwana M. Interleukin-6 as a potential therapeutic target for pulmonary arterial hypertension. Int J Rheumatol. 2010;2010:720305.

33. Müllberg J, et al. The soluble human IL-6 receptor. Mutational characterization of the proteolytic cleavage site. J Immunol. 1994;152(10):4958-4968.

34. Esparza-Gordillo J, et al. A functional IL-6 receptor (IL6R) variant is a risk factor for persistent atopic dermatitis. J Allergy Clin Immunol. 2013;132(2):371-377.

35. Marinou I, Walters K, Winfield J, Bax DE, Wilson AG. A gain of function polymorphism in the interleukin 6 receptor influences RA susceptibility. Ann Rheum Dis. 2010;69(6):1191-1194.

36. Ferreira MA, et al. Identification of IL6R and chromosome 11q13.5 as risk loci for asthma. Lancet. 2011;378(9795):1006-1014.

37. Interleukin-6 Receptor Mendelian Randomisation Analysis (IL6R MR) Consortium, et al. The interleukin-6 receptor as a target for prevention of coronary heart disease: a mendelian randomisation analysis. Lancet. 2012;379(9822):1214-1224.

38. Szpakowicz A, et al. The rs2228145 polymorphism in the interleukin- 6 receptor and its association with long-term prognosis after myocardial infarction in a pilot study. Arch Med Sci. 2017;13(1):93-99.

39. Gigante B, et al. Analysis of the role of interleukin 6 receptor haplotypes in the regulation of circulating levels of inflammatory biomarkers and risk of coronary heart disease. PLOS ONE. 2015;10(3):e0119980.

40. Guignabert C, et al. Tie2-mediated loss of peroxisome proliferator-activated receptor-gamma in mice causes PDGF receptor-beta-dependent pulmonary arterial muscularization. Am JPhysiol Lung Cell Mol Physiol. 2009;297(6):L1082-L1090.

41. Guignabert C, et al. Transgenic mice overexpressing the 5-hydroxytryptamine transporter gene in smooth muscle develop pulmonary hypertension. Circ Res. 2006;98(10):1323-1330.

42. Huertas A, et al. Leptin signalling system as a target for pulmonary arterial hypertension therapy. Eur Respir J. 2015;45(4):1066-1080.

43. Tu L, et al. A critical role for p130Cas in the progression of pulmonary hypertension in humans and rodents. Am J Respir Crit Care Med. 2012;186(7):666-676.

44. Tu L, et al. Autocrine fibroblast growth factor-2 signaling contributes to altered endothelial phenotype in pulmonary hypertension. Am J Respir Cell Mol Biol. 2011;45(2):311-322.

45. El-Bizri N, et al. Smooth muscle protein 22alpha-mediated patchy deletion of Bmpr1a impairs cardiac contractility but protects against pulmonary vascular remodeling. Circ Res. 2008;102(3):380-388.

46. Guignabert C, et al. Dasatinib induces lung vascular toxicity and predisposes to pulmonary hypertension. JClin Invest. 2016;126(9):3207-3218.

47. de Man FS, et al. Dysregulated renin-angiotensinaldosterone system contributes to pulmonary arterial hypertension. Am J Respir Crit Care Med. 2012;186(8):780-789.

48. Eddahibi S, et al. Cross talk between endothelial and smooth muscle cells in pulmonary hypertension: critical role for serotonininduced smooth muscle hyperplasia. Circulation. 2006;113(15):1857-1864.

49. El-Bizri N, et al. SM22alpha-targeted deletion of bone morphogenetic protein receptor $1 \mathrm{~A}$ in mice impairs cardiac and vascular development, and influences organogenesis. Development. 2008;135(17):2981-2991.

50. Wang Y, et al. Overexpression of the insulin-like growth factor II receptor increases $\beta$-amyloid production and affects cell viability. Mol Cell Biol. 2015;35(14):2368-2384.

51. Zhu Y, et al. Caspase cleavage of cytochrome $\mathrm{c} 1$ disrupts mitochondrial function and enhances cytochrome c release. Cell Res. 2012;22(1):127-141.

52. Long L, et al. Selective enhancement of endothelial BMPR-II with BMP9 reverses pulmonary arterial hypertension. Nat Med. 2015;21(7):777-785. 